\title{
RANDOMIZED OBSERVATION PERIODS FOR THE COMPOUND POISSON RISK MODEL: THE DISCOUNTED PENALTY FUNCTION
}

\author{
Hansjörg Albrecher; Eric C.K. Cheung ${ }^{\dagger}$ and Stefan Thonhauser*
}

\begin{abstract}
In the framework of collective risk theory, we consider a compound Poisson risk model for the surplus process where the process (and hence ruin) can only be observed at random observation times. For Erlang $(n)$ distributed inter-observation times, explicit expressions for the discounted penalty function at ruin are derived. The resulting model contains both the usual continuous-time and the discrete-time risk model as limiting cases, and can be used as an effective approximation scheme for the latter. Numerical examples are given that illustrate the effect of random observation times on various ruin-related quantities.
\end{abstract}

Keywords: Compound Poisson risk model, Gerber-Shiu function, Erlangization, defective renewal equation, discounted density

\section{Introduction}

In the classical Cramér-Lundberg risk model, the surplus process of an insurance portfolio $\{C(t)\}_{t \geq 0}$ is given by

$$
C(t):=x+c t-S(t)=x+c t-\sum_{i=1}^{N(t)} Y_{i}, \quad t \geq 0,
$$

where $x=C(0) \geq 0$ is the initial surplus level, $c>0$ is the constant premium income per unit time, and the aggregate claims process $\{S(t)\}_{t \geq 0}$ is a compound Poisson process, comprising a homogeneous Poisson process $\{N(t)\}_{t \geq 0}$ with rate $\lambda>0$, and independent and identically distributed (i.i.d.) claim sizes $\left\{Y_{i}\right\}_{i=1}^{\infty}$ that are independent of $\{N(t)\}_{t \geq 0}$ and distributed as a generic continuous random variable (r.v.) $Y$ with c.d.f. $F_{Y}(\cdot)$, density $f_{Y}(\cdot)$ and corresponding Laplace transform $\widetilde{f}_{Y}(s)=\int_{0}^{\infty} e^{-s y} f_{Y}(y) d y$. Ruin of the risk process is the event that $C(t)<0$ for some $t \geq 0$.

In this classical model it is possible to observe the current value of the surplus (and hence also to observe possible ruin) continuously. In practice, however, it may be more reasonable to assume that the balance of the books is only checked on a periodic basis, which naturally leads to the study of discrete-time risk models (see e.g. Asmussen \& Albrecher [2] for a recent survey). However, discrete-time risk models have the distinctive disadvantage that they usually do not lead to explicit expressions for the ruin

\footnotetext{
${ }^{*}$ Department of Actuarial Science, Faculty of Business and Economics, University of Lausanne, UNIL-Dorigny, CH-1015 Lausanne, Switzerland and Swiss Finance Institute.

${ }^{\dagger}$ Department of Statistics and Actuarial Science, University of Hong Kong, Pokfulam, Hong Kong.
} 
probability and related quantities. Therefore, we propose in this paper to use a computational bridge between the continuous-time and the discrete-time collective risk models that still enables explicit expressions. This will turn out to serve as a quite satisfying approximation of the discrete-time counterpart.

Concretely, assume that the compound Poisson risk model (1.1) can only be observed at random times $\left\{Z_{k}\right\}_{k=0}^{\infty}$ (i.e., $Z_{k}$ is the $k$-th observation time, with $Z_{0}=0$ ). Let $T_{k}=Z_{k}-Z_{k-1}$ be the $k$-th inter-observation time and assume that $\left\{T_{k}\right\}_{k=1}^{\infty}$ is an i.i.d. sequence with generic r.v. $T$, independent of the claim number process $\{N(t)\}_{t \geq 0}$ and the claim sizes $\left\{Y_{i}\right\}_{i=1}^{\infty}$. Letting $U(k)=C\left(Z_{k}\right)$, the resulting process $\{U(k)\}_{k=0}^{\infty}$ can be described recursively by

$$
U(k)=U(k-1)+c T_{k}-\left[S\left(Z_{k}\right)-S\left(Z_{k-1}\right)\right], \quad k=1,2, \ldots,
$$

with initial surplus $U(0)=C(0)=x$. Then the time of ruin in this modified model is $\tau=Z_{k^{*}}$, where $k^{*}=\inf \{k \geq 1: U(k)<0\}$. Note that if the surplus of (1.1) becomes negative, but is again positive at the next observation, the process is not ruined (see e.g. the sample path in Figure 1, where ruin is only declared at $\left.t=Z_{5}\right)$. One may interpret the random walk $\{U(k)\}_{k=0}^{\infty}$ as the one embedded in a dependent Sparre Andersen risk model with generic 'interclaim time' $T$ and 'claim size' $\sum_{i=1}^{N(T)} Y_{i}$ (see Cheung et al. [6]). The safety loading condition for this model is - as in the original continuous-time risk model - still $c>\lambda \mathbb{E}[Y]$.

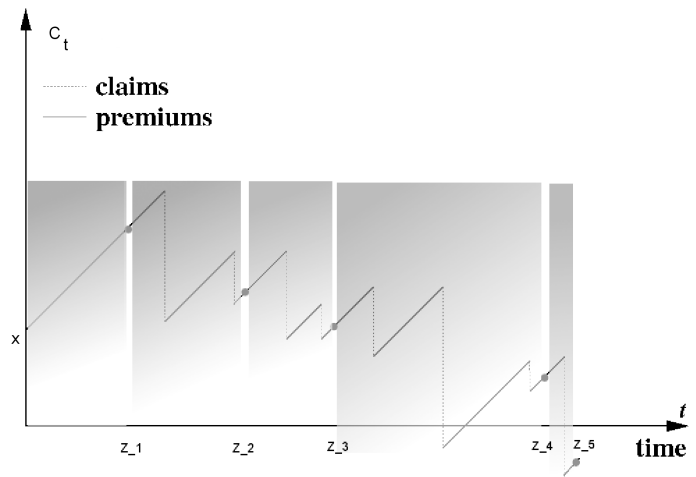

Figure 1: The value of the compound Poisson surplus process is only observed at random times $Z_{k}(k=0,1, \ldots)$

For the purpose of this paper, we assume $T$ to be $\operatorname{Erlang}(n)$ distributed with density

$$
f_{T}(t):=\frac{\gamma^{n} t^{n-1} e^{-\gamma t}}{(n-1) !}, \quad t>0, \gamma>0
$$

and corresponding Laplace transform $\tilde{f}_{T}(s)=[\gamma /(\gamma+s)]^{n}$. The case $n=1$ refers to exponentially distributed observation intervals, i.e. lack of memory of the time until the next observation. If, on the other hand, ones fixes $\mathbb{E}[T]=h$ and chooses $n$ sufficiently large, this approximates the discrete-time risk model with deterministic time steps $h$, since the Erlang distribution for $n \rightarrow \infty$ and fixed expected value $\mathbb{E}[T]=h$ converges in distribution to a point mass in $h$. Finally, if $\gamma \rightarrow \infty$ for fixed $n$, then $T$ converges in distribution to a point mass at 0 , and this limit corresponds to the classical continuous-time risk model $\{C(t)\}_{t \geq 0}$ described by (1.1) (i.e. continuous observation of the process and monitoring of potential ruin). 
Note that for the calculation of finite-time ruin probabilities in the continuous model (1.1), a randomization of the time horizon by an Erlang r.v. (called Erlangization) was successfully employed in Asmussen et al. [3] (see also Ramaswami et al. [14] and Stanford et al. [15, 16] for extensions).

In this paper, the Erlangization is used on another level, namely for each inter-observation time $T_{k}$ of the risk process. The focus will be on the expected discounted penalty function at ruin, introduced by Gerber \& Shiu [10], which comprises information on the time of ruin, the surplus prior to ruin and the deficit at ruin. The natural adaption of this function in our context is

$$
m_{\delta}(x):=\mathbb{E}\left[e^{-\delta \tau} w\left(U\left(k^{*}-1\right),\left|U\left(k^{*}\right)\right|\right) I_{\{\tau<\infty\}} \mid U(0)=x\right], \quad x \geq 0 .
$$

As usual, $\delta \geq 0$ can be interpreted as a force of interest or as the Laplace transform argument with respect to the ruin time $\tau, I_{\{\cdot\}}$ is the indicator function and $w\left(x_{1}, x_{2}\right)$ is a penalty function depending on the surplus prior to ruin $U\left(k^{*}-1\right)$ and the deficit at ruin $\left|U\left(k^{*}\right)\right|$. Throughout, we assume that $w(\cdot, \cdot)$ satisfies some mild integrability conditions such that the expectation in (1.2) exists. Since $U\left(k^{*}-1\right)$ corresponds to the surplus immediately after the second-last claim before ruin in the afore-mentioned dependent Sparre Andersen risk model (see Badescu et al. [4, Section 5] and Cheung et al. [5]), various structural properties derived in Cheung et al. [6] can be applied to the study of $m_{\delta}(x)$, and at the same time help to carry over some of the properties of the classical continuous-time model to the discretized version. In the sequel paper [1], we will investigate the effects of random observation times on the performance of dividend strategies.

The remainder of the paper is organized as follows. Section 2 studies Gerber-Shiu functions for which the penalty function only depends on the deficit at ruin (i.e. $w\left(x_{1}, x_{2}\right) \equiv w_{2}\left(x_{2}\right)$ ). For exponential interobservation time $T$ and exponential claim size $Y$, we exploit the Markov structure of the resulting risk process to derive integro-differential equations (IDEs) for $m_{\delta}(\cdot)$, which can then be reduced to differential equations. In Section 3 we characterize the Gerber-Shiu function (1.2) in a general model framework as the solution of a defective renewal equation. The discounted density of the increment between successive observations plays an important role in the analysis. Section 4 treats this discounted increment density in detail for claim size distributions with rational Laplace transform and gives explicit expressions for $m_{\delta}(\cdot)$ for penalty functions of the type $w\left(x_{1}, x_{2}\right) \equiv w_{2}\left(x_{2}\right)$. Finally, numerical examples are given in Section 5 to illustrate the effect of random observations on some ruin-related quantities.

\section{Method of integro-differential equations when $w\left(x_{1}, x_{2}\right) \equiv w_{2}\left(x_{2}\right)$}

In this section, we consider the Gerber-Shiu function (1.2) for the case where the penalty function depends on the ruin deficit only, i.e. $w\left(x_{1}, x_{2}\right) \equiv w_{2}\left(x_{2}\right)$, where $w_{2}\left(x_{2}\right)$ is a continuous function for $x_{2} \geq 0$. If the inter-observation times are exponentially distributed, then the process is Markovian and we will restrict our focus in this section on that case. For more general inter-observation time distributions see Remark 2.2 below.

\subsection{IDEs for exponential inter-observation times}

With observations and claims arriving (independently) at Poisson rates $\gamma$ and $\lambda$ respectively, we consider a time interval $(0, h)$ and condition on whether an observation time occurs in this interval before a claim 
occurs, or a claim occurs before an observation time occurs, or neither a claim nor an observation time occurs until time $h$. We then arrive at

$$
\begin{aligned}
m_{\delta}(x)= & e^{-(\delta+\lambda+\gamma) h} m_{\delta}(x+c h)+\int_{0}^{h} e^{-(\lambda+\delta) t} \gamma e^{-\gamma t}\left[m_{\delta}(x+c t) I_{\{x+c t \geq 0\}}+w_{2}(-(x+c t)) I_{\{x+c t<0\}}\right] d t \\
& +\int_{0}^{h} e^{-(\delta+\gamma) t} \lambda e^{-\lambda t} \int_{0}^{\infty} m_{\delta}(x+c t-y) f_{Y}(y) d y d t, \quad x \in \mathbb{R}
\end{aligned}
$$

Note that if a claim occurs before an observation time, it is possible for the surplus level to drop below zero without being observed. Therefore, the domain of $m_{\delta}(x)$ has been extended to $x \in \mathbb{R}$, even if eventually one usually will declare time 0 an observation time. The right-continuity of $m_{\delta}(x)$ on $\mathbb{R}$, in particular in $x=0$, is established by letting $h \rightarrow 0$, because the integrands are (locally) bounded. Taking the derivative of (2.1) with respect to $h$ and letting $h \rightarrow 0$, we obtain the system of IDEs

$$
\begin{aligned}
& 0=c m_{\delta}^{\prime}(x)-(\lambda+\gamma+\delta) m_{\delta}(x)+\gamma w_{2}(-x)+\lambda \int_{0}^{\infty} m_{\delta}(x-y) f_{Y}(y) d y, \quad x<0, \\
& 0=c m_{\delta}^{\prime}(x)-(\lambda+\delta) m_{\delta}(x)+\lambda \int_{0}^{\infty} m_{\delta}(x-y) f_{Y}(y) d y, \quad x \geq 0 .
\end{aligned}
$$

A priori, the derivatives above are right-hand derivatives, but by starting the same conditioning argument at some point $x-c h$ for small $h>0$, one not only establishes the left-continuity of $m_{\delta}($.$) on \mathbb{R}$ but also finds that the left-hand derivatives of $m_{\delta}(\cdot)$ still fulfill (2.2) and (2.3). Consequently, $m_{\delta}(x)$ is indeed differentiable for $x \in \mathbb{R} \backslash\{0\}$, and at $x=0$ we have

$$
c m_{\delta}^{\prime}(0-)-c m_{\delta}^{\prime}(0+)=\gamma m_{\delta}(0)-\gamma w_{2}(0),
$$

so that in general $m_{\delta}(x)$ is not differentiable at $x=0$.

Let us rewrite $m_{\delta}(x)$ as

$$
m_{\delta}(x):= \begin{cases}m_{\delta, L}(x), & x<0, \\ m_{\delta, U}(x), & x \geq 0,\end{cases}
$$

where the extra subscripts ' $L$ ' and ' $U$ ' stand for 'lower' and 'upper' layer, respectively. Then the IDEs (2.2) and (2.3) can be rewritten as

$$
\begin{aligned}
& 0=c m_{\delta, L}^{\prime}(x)-(\lambda+\gamma+\delta) m_{\delta, L}(x)+\gamma w_{2}(-x)+\lambda \int_{0}^{\infty} m_{\delta, L}(x-y) f_{Y}(y) d y, \quad x<0, \\
& 0=c m_{\delta, U}^{\prime}(x)-(\lambda+\delta) m_{\delta, U}(x)+\lambda \int_{0}^{x} m_{\delta, U}(x-y) f_{Y}(y) d y+\lambda \int_{x}^{\infty} m_{\delta, L}(x-y) f_{Y}(y) d y, \quad x \geq 0 .
\end{aligned}
$$

For a complete characterization of the solution of the above system, one can use the continuity condition

$$
m_{\delta, L}(0-)=m_{\delta, U}(0+),
$$

as well as the boundary conditions for $\lim _{x \rightarrow-\infty} m_{\delta, L}(x)$ and $\lim _{x \rightarrow \infty} m_{\delta, U}(x)$ which depend on the form of the penalty function $w_{2}(\cdot)$. The next subsection shows how this can be carried out in the case of exponential claims. 


\subsection{Constructing a solution - the exponential claim case}

Assume that the claim size density is given by $f_{Y}(y)=\nu e^{-\nu y}, y>0$, for some parameter $\nu>0$. We proceed by applying the operator $(d / d x+\nu)$ to (2.4) and (2.5), respectively. For the lower layer $x<0$, this leads to

$$
c m_{\delta, L}^{\prime \prime}(x)+[c \nu-(\lambda+\gamma+\delta)] m_{\delta, L}^{\prime}(x)-(\gamma+\delta) \nu m_{\delta, L}(x)=\gamma w_{2}^{\prime}(-x)+\gamma \nu w_{2}(-x), \quad x<0,
$$

where we assume that $w_{2}(\cdot)$ is differentiable. This second-order differential equation with constant coefficients has characteristic equation (in $\xi$ )

$$
\xi^{2}+\left(\nu-\frac{\lambda+\gamma+\delta}{c}\right) \xi-\frac{(\gamma+\delta) \nu}{c}=0
$$

which has a positive root $\rho_{\gamma}>0$ and a negative root $-R_{\gamma}<0$. Note that the above equation is equivalent to the usual Lundberg's fundamental equation in the classical model with continuous observation when $\gamma=0$. The solution of (2.7) will now depend on the nature of the penalty function $w_{2}(\cdot)$ involved in the inhomogeneous term. For the upper layer $x \geq 0$, the same procedure leads to a second-order homogeneous differential equation for $m_{\delta, U}(x)$. The latter also has characteristic equation (2.8) but with $\gamma=0$, and the resulting roots are denoted by $\rho_{0} \geq 0$ and $-R_{0}<0$. Hence, one has

$$
m_{\delta, U}(x)=A_{1} e^{\rho_{0} x}+A_{2} e^{-R_{0} x}, \quad x \geq 0,
$$

for some constants $A_{1}, A_{2}$.

Let us now choose the penalty function $w_{2}\left(x_{2}\right)=e^{-r_{2} x_{2}}$ for $r_{2} \geq 0$, so that $m_{\delta}(x)$ corresponds to the bivariate Laplace transform of the time of ruin $\tau$ and the deficit at ruin $\left|U\left(k^{*}\right)\right|$ (clearly, this choice of penalty function is not very restrictive). Then the inhomogeneous term in (2.7) is proportional to $e^{r_{2} x}$ and the solution $m_{\delta, L}(x)$ will be of the form

$$
m_{\delta, L}(x)=C_{1} e^{\rho_{\gamma} x}+C_{2} e^{-R_{\gamma} x}+C_{3} e^{r_{2} x}, \quad x \leq 0,
$$

for some constants $C_{1}, C_{2}, C_{3}$. Note that both $\nu-R_{\gamma}$ and $\nu-R_{0}$ are positive and therefore the integrals in (2.4) and (2.5) exist.

The coefficients in (2.9) and (2.10) can now be determined by exploiting the boundary conditions at $-\infty$ and $+\infty$. First, under positive safety loading (or, alternatively, whenever $\delta>0$ ), the bound $m_{\delta, U}(x) \leq E\left[e^{-\delta \tau} I_{\{\tau<\infty\}} \mid U(0)=x\right]$ for $x \geq 0$ implies the natural condition $\lim _{x \rightarrow \infty} m_{\delta, U}(x)=0$. An immediate consequence is that $A_{1}=0$, since $\rho_{0} \geq 0$. Next, the condition at $x \rightarrow-\infty$ depends on whether $r_{2}=0$ or $r_{2}>0$. If $r_{2}=0$, then $m_{\delta}(x)$ is simply the Laplace transform of the time of ruin. But as $x \rightarrow-\infty$, ruin occurs (almost surely) at the time of the first observation $Z_{1}=T_{1}$ which is exponential with mean $1 / \gamma$. In contrast, if $r_{2}>0, x \rightarrow-\infty$ implies infinite deficit at ruin, so that $m_{\delta, L}(x)$ tends to 0. Thus,

$$
\lim _{x \rightarrow-\infty} m_{\delta, L}(x)= \begin{cases}\mathbb{E}\left[e^{-\delta T}\right]=\frac{\gamma}{\gamma+\delta}, & r_{2}=0 \\ 0, & r_{2}>0 .\end{cases}
$$

The boundedness of $\lim _{x \rightarrow-\infty} m_{\delta, L}(x)$ and $R_{\gamma}>0$ imply that $C_{2}=0$. To determine the remaining constants $A_{2}, C_{1}$ and $C_{3}$, we substitute (2.9) and (2.10) into the IDEs (2.4) and (2.5). For (2.4), equating the coefficients of $e^{\rho_{\gamma} x}$ does not yield any information, whereas comparing the coefficients of $e^{r_{2} x}$ gives

$$
C_{3}=-\frac{\gamma}{c r_{2}-(\lambda+\gamma+\delta)+\lambda \frac{\nu}{\nu+r_{2}}} .
$$


Similarly, for (2.5), the coefficients of $e^{-R_{0} x}$ lead to no extra information, whereas the coefficients of $e^{-\nu x}$ imply

$$
A_{2} \frac{1}{\nu-R_{0}}=C_{1} \frac{1}{\nu+\rho_{\gamma}}+C_{3} \frac{1}{\nu+r_{2}}
$$

Finally, the continuity condition $(2.6)$ in $x=0$ gives

$$
A_{2}=C_{1}+C_{3},
$$

and the system of linear equations (2.11) and (2.12) can easily be solved to give $A_{2}$ and $C_{1}$. Altogether, we arrive at

$$
m_{\delta, L}(x)=\frac{\gamma\left(\frac{1}{\nu+r_{2}}-\frac{1}{\nu-R_{0}}\right) e^{\rho_{\gamma} x}}{\left(c r_{2}-(\lambda+\gamma+\delta)+\lambda \frac{\nu}{\nu+r_{2}}\right)\left(\frac{1}{\nu+\rho_{\gamma}}-\frac{1}{\nu-R_{0}}\right)}-\frac{\gamma e^{r_{2} x}}{c r_{2}-(\lambda+\gamma+\delta)+\lambda \frac{\nu}{\nu+r_{2}}}, \quad x \leq 0
$$

and

$$
m_{\delta, U}(x)=\frac{\gamma\left(\frac{1}{\nu+r_{2}}-\frac{1}{\nu+\rho_{\gamma}}\right) e^{-R_{0} x}}{\left(c r_{2}-(\lambda+\gamma+\delta)+\lambda \frac{\nu}{\nu+r_{2}}\right)\left(\frac{1}{\nu+\rho_{\gamma}}-\frac{1}{\nu-R_{0}}\right)}, \quad x \geq 0
$$

respectively. In view of the identity

$$
c r_{2}-(\lambda+\gamma+\delta)+\lambda \frac{\nu}{\nu+r_{2}}=\frac{c\left(r_{2}-\rho_{\gamma}\right)\left(r_{2}+R_{\gamma}\right)}{\nu+r_{2}}
$$

these formulas simplify to

$$
\begin{aligned}
m_{\delta, L}(x)= & \frac{\gamma}{c\left(r_{2}-\rho_{\gamma}\right)\left(r_{2}+R_{\gamma}\right)}\left(\frac{\left(\nu+\rho_{\gamma}\right)\left(r_{2}+R_{0}\right)}{\rho_{\gamma}+R_{0}} e^{\rho_{\gamma} x}-\left(\nu+r_{2}\right) e^{r_{2} x}\right) \\
= & \frac{\gamma\left(\nu+\rho_{\gamma}\right)}{c\left(\rho_{\gamma}+R_{\gamma}\right)}\left(\frac{1}{r_{2}-\rho_{\gamma}}+\frac{R_{\gamma}-R_{0}}{\left(\rho_{\gamma}+R_{0}\right)\left(r_{2}+R_{\gamma}\right)}\right) e^{\rho_{\gamma} x} \\
& -\frac{\gamma}{c\left(\rho_{\gamma}+R_{\gamma}\right)}\left(\frac{\nu+\rho_{\gamma}}{r_{2}-\rho_{\gamma}}-\frac{\nu-R_{\gamma}}{r_{2}+R_{\gamma}}\right) e^{r_{2} x}, \quad x \leq 0,
\end{aligned}
$$

and

$$
\begin{aligned}
m_{\delta, U}(x) & =\frac{\gamma\left(\nu-R_{0}\right)}{c\left(\rho_{\gamma}+R_{0}\right)\left(r_{2}+R_{\gamma}\right)} e^{-R_{0} x} \\
& =\frac{R_{\gamma}-R_{0}}{r_{2}+R_{\gamma}} e^{-R_{0} x}, \quad x \geq 0 .
\end{aligned}
$$

The Laplace transform inversion with respect to $r_{2}$ of (2.13) and (2.14) will give the discounted density of the deficit at ruin $h_{\delta, L}^{*}(y \mid x)$, i.e.

$$
m_{\delta, L}(x)=\int_{0}^{\infty} e^{-r_{2} y} h_{\delta, L}^{*}(y \mid x) d y, \quad x<0 \quad \text { and } \quad m_{\delta, U}(x)=\int_{0}^{\infty} e^{-r_{2} y} h_{\delta, U}^{*}(y \mid x) d y, \quad x \geq 0
$$


with

$$
\begin{aligned}
h_{\delta, L}^{*}(y \mid x)= & \frac{\gamma\left(\nu+\rho_{\gamma}\right)}{c\left(\rho_{\gamma}+R_{\gamma}\right)}\left(e^{\rho_{\gamma} y}+\frac{R_{\gamma}-R_{0}}{\rho_{\gamma}+R_{0}} e^{-R_{\gamma} y}\right) e^{\rho_{\gamma} x} \\
& -\frac{\gamma}{c\left(\rho_{\gamma}+R_{\gamma}\right)}\left[\left(\nu+\rho_{\gamma}\right) e^{\rho_{\gamma}(x+y)}-\left(\nu-R_{\gamma}\right) e^{-R_{\gamma}(x+y)}\right] I_{\{y>-x\}} \\
= & \frac{\gamma\left(\nu+\rho_{\gamma}\right)\left(R_{\gamma}-R_{0}\right)}{c\left(\rho_{\gamma}+R_{\gamma}\right)\left(\rho_{\gamma}+R_{0}\right)} e^{\rho_{\gamma} x-R_{\gamma} y}+\frac{\gamma\left(\nu+\rho_{\gamma}\right)}{c\left(\rho_{\gamma}+R_{\gamma}\right)} e^{\rho_{\gamma}(x+y)} I_{\{y \leq-x\}} \\
& +\frac{\gamma\left(\nu-R_{\gamma}\right)}{c\left(\rho_{\gamma}+R_{\gamma}\right)} e^{-R_{\gamma}(x+y)} I_{\{y>-x\}}, \quad y>0,
\end{aligned}
$$

and

$$
h_{\delta, U}^{*}(y \mid x)=\left(R_{\gamma}-R_{0}\right) e^{-R_{0} x-R_{\gamma} y}, \quad y>0 .
$$

Remark 2.1 It is instructive to note that having identified the discounted densities in (2.16) and (2.17), the Gerber-Shiu functions $m_{\delta, L}(x)$ and $m_{\delta, U}(x)$ for an arbitrary penalty function $w_{2}(\cdot)$ are given by $(2.15)$ simply with $e^{-r_{2} y}$ replaced by $w_{2}(y)$.

Example 2.1 The Laplace transform of the time to ruin is obtained for $r_{2}=0$, and so we get from (2.13) and (2.14)

$$
\mathbb{E}\left[e^{-\delta \tau} I_{\{\tau<\infty\}} \mid U(0)=x\right]=\left(1-\frac{R_{0}}{R_{\gamma}}\right) e^{-R_{0} x} I_{\{x \geq 0\}}+\frac{\gamma}{c \rho_{\gamma} R_{\gamma}}\left(\nu-\frac{\nu+\rho_{\gamma}}{1+\rho_{\gamma} / R_{0}} e^{\rho_{\gamma} x}\right) I_{\{x<0\}}
$$

For $\gamma \rightarrow \infty$, we have $\rho_{\gamma} \rightarrow \infty, R_{\gamma} \rightarrow \nu$ and $\rho_{\gamma} / \gamma \rightarrow 1 / c$ (by Vieta's rule for (2.8)), so that we indeed obtain the formula

$$
\mathbb{E}\left[e^{-\delta \tau_{C L}} I_{\left\{\tau_{C L}<\infty\right\}} \mid C(0)=x\right]=\left(1-\frac{R_{0}}{\nu}\right) e^{-R_{0} x} I_{\{x \geq 0\}}+I_{\{x<0\}}
$$

for the classical compound Poisson risk model with continuous observation in the limit (see e.g. Gerber \& Shiu (1998)). Note that for $\delta=0$ we further have $R_{0}=\nu-\lambda / c$ and the formula further simplifies to the classical ruin probability

$$
\psi_{C L}(x)=\frac{\lambda}{\nu c} e^{-(\nu-\lambda / c) x} I_{\{x \geq 0\}}+I_{\{x<0\}} .
$$

Example 2.2 The expected discounted deficit at ruin is obtained from (2.13) and (2.14) by taking the (negative) derivative with respect to $r_{2}$ at $r_{2}=0$. Together with Vieta's rule this leads to

$$
\begin{aligned}
\mathbb{E}\left[e^{-\delta \tau}\left|U\left(k^{*}\right)\right| I_{\{\tau<\infty\}} \mid U(0)=x\right]= & I_{\{x \geq 0\}} \frac{R_{\gamma}-R_{0}}{R_{\gamma}^{2}} e^{-R_{0} x} \\
& +I_{\{x<0\}} \frac{\gamma\left(\nu+\rho_{\gamma}\right)}{c\left(\rho_{\gamma}+R_{\gamma}\right)}\left(\frac{1}{\rho_{\gamma}^{2}}-\frac{R_{\gamma}-R_{0}}{\left(\rho_{\gamma}+R_{0}\right) R_{\gamma}^{2}}\right) e^{\rho_{\gamma} x} \\
& -I_{\{x<0\}}\left(\frac{\gamma(c-\lambda / \nu)}{(\gamma+\delta)^{2}}+\frac{\gamma}{\gamma+\delta} x\right) .
\end{aligned}
$$


Note that the same result can also be derived by multiplying the discounted densities in (2.16) and (2.17) by $y$ and then integrating with respect to $y$ (see Remark 2.1). By the same limit operation as in Example 2.1 , we obtain for $\gamma \rightarrow \infty$

$$
\mathbb{E}\left[e^{-\delta \tau_{C L}}\left|C\left(\tau_{C L}\right)\right| I_{\left\{\tau_{C L}<\infty\right\}} \mid C(0)=x\right]=\frac{\nu-R_{0}}{\nu^{2}} e^{-R_{0} x} I_{\{x \geq 0\}}-x I_{\{x<0\}},
$$

which is the expected discounted deficit at ruin for the classical compound Poisson risk model with continuous observation (see again Gerber \& Shiu (1998)).

Remark 2.2 In principle, the method of this section can be extended to the case of phase-type observation intervals (as well as more complicated claim size distributions), by increasing the dimension of the state space (and hence regaining the Markovian structure in this new state space). However, this leads to considerable effort, since one has to keep track of two layers for each of the $n$ phases which results in $2 n$ interacting IDEs in the case of $\operatorname{Erlang}(n)$ intervals. In the next section, we discuss an alternative approach that not only avoids these difficulties but also allows for the study of the general Gerber-Shiu function (1.2) including the surplus prior to ruin.

\section{Defective renewal equation and discounted increments}

\subsection{A defective renewal equation for $m_{\delta}(x)$}

Recall from Section 1 that the sequence $\{U(k)\}_{k=0}^{\infty}$ can be interpreted as surplus levels after each claim in a dependent Sparre Andersen risk model with generic 'interclaim time' $T$ and 'claim size' $\sum_{i=1}^{N(T)} Y_{i}$. Since the surplus prior to ruin $U\left(k^{*}-1\right)$ in the present model corresponds to the surplus after the second-last claim before ruin in that dependent Sparre Andersen risk model, we can utilize some results of Cheung et al. [6] for our purpose.

Given $U(0)=x$, the form of the discounted joint density of the pair $\left(U\left(k^{*}-1\right),\left|U\left(k^{*}\right)\right|\right)$ depends on whether ruin occurs at the first positive observation time $Z_{1}$ or not. For ruin at time $Z_{1}$, one has the deterministic relationship $U\left(k^{*}-1\right)=U(0)=x$ and only requires the discounted density of $\left|U\left(k^{*}\right)\right|$ at $y$, which we denote by $h_{1, \delta}^{*}(y \mid x)$. On the other hand, for ruin after $Z_{1}$, the discounted joint density of $\left(U\left(k^{*}-1\right),\left|U\left(k^{*}\right)\right|\right)$ at $(z, y)$ is denoted by $h_{2, \delta}^{*}(z, y \mid x)$. The Gerber-Shiu function $m_{\delta}(x)$ defined by $(1.2)$ can now be represented as

$$
m_{\delta}(x)=\int_{0}^{\infty} w(x, y) h_{1, \delta}^{*}(y \mid x) d y+\int_{0}^{\infty} \int_{0}^{\infty} w(z, y) h_{2, \delta}^{*}(z, y \mid x) d z d y, \quad x \geq 0,
$$

and the discounted density of the deficit at ruin is

$$
h_{\delta}^{*}(y \mid x)=h_{1, \delta}^{*}(y \mid x)+\int_{0}^{\infty} h_{2, \delta}^{*}(z, y \mid x) d z, \quad y>0,
$$

which corresponds to $h_{\delta, U}^{*}(y \mid x)$ of the previous section. Then, by considering the first drop in surplus below its initial level in the associated dependent Sparre Andersen risk model, one has

$$
\begin{aligned}
m_{\delta}(x)= & \int_{0}^{x} m_{\delta}(x-y) h_{\delta}^{*}(y \mid 0) d y+\int_{x}^{\infty} w(x, y-x) h_{1, \delta}^{*}(y \mid 0) d y \\
& +\int_{x}^{\infty} \int_{0}^{\infty} w(z+x, y-x) h_{2, \delta}^{*}(z, y \mid 0) d z d y, \quad x \geq 0 .
\end{aligned}
$$


Because of $\int_{0}^{\infty} h_{\delta}^{*}(y \mid 0) d y=\mathbb{E}\left[e^{-\delta \tau} I_{\{\tau<\infty\}} \mid U(0)=0\right]<1$ under $\delta>0$ (or under positive safety loading), this is a defective renewal equation (for its solution, see e.g. Willmot \& Lin [18, p.154]). Hence, once the discounted densities $h_{1, \delta}^{*}(\cdot \mid 0)$ and $h_{2, \delta}^{*}(\cdot, \cdot \mid 0)$ are determined, the Gerber-Shiu function $m_{\delta}(\cdot)$ can be determined in full generality. Section 3.3 will deal with expressions for $h_{1, \delta}^{*}(\cdot \mid 0)$ and $h_{2, \delta}^{*}(\cdot, \cdot \mid 0)$ for $\operatorname{Erlang}(n)$ distributed observation intervals.

\subsection{Discounted increments between successive observations}

Consider now Erlang $(n)$ distributed observation intervals and an arbitrary claim size distribution. Let us return to the embedded random walk interpretation for $\{U(k)\}_{k=0}^{\infty}$. Concretely, the pairs $\left(T_{k}, U(k-\right.$ $1)-U(k))(k=1,2, \ldots)$ form an i.i.d. sequence with generic distribution $\left(T, \sum_{i=1}^{N(T)} Y_{i}-c T\right)$ and joint Laplace transform

$$
\begin{aligned}
\mathbb{E}\left[e^{-\delta T-s\left(\sum_{i=1}^{N(T)} Y_{i}-c T\right)}\right] & =\mathbb{E}\left[e^{-(\delta-c s) T} \mathbb{E}\left[e^{-s \sum_{i=1}^{N(T)} Y_{i}} \mid T\right]\right]=\mathbb{E}\left[e^{-\left\{(\delta-c s) T+\lambda\left[1-\widetilde{f}_{Y}(s)\right] T\right\}}\right] \\
& =\left(\frac{\gamma}{\gamma+\delta-c s+\lambda\left[1-\widetilde{f}_{Y}(s)\right]}\right)^{n} .
\end{aligned}
$$

On the other hand, one may also write

$$
\mathbb{E}\left[e^{-\delta T-s\left(\sum_{i=1}^{N(T)} Y_{i}-c T\right)}\right]=\int_{-\infty}^{\infty} e^{-s y} g_{\delta}(y) d y,
$$

where $g_{\delta}(y)$ represents the discounted density of the increment $\sum_{i=1}^{N(T)} Y_{i}-c T$ between successive observation times. It will be useful to decompose $g_{\delta}(y)$ into

$$
g_{\delta}(y)=g_{\delta,-}(-y) I_{\{y<0\}}+g_{\delta,+}(y) I_{\{y>0\}}, \quad-\infty<y<\infty .
$$

Remark 3.1 To give a formal definition of the above discounted densities, consider the increment $\sum_{i=1}^{N(t)} Y_{i}-c t$ from time 0 to time $t$ (with $t>0$ being fixed). Note that the distribution of $\sum_{i=1}^{N(t)} Y_{i}-c t$ has a point mass at $-c t$ with probability $e^{-\lambda t}$ (for the case when there is no claim within $[0, t]$ ). Denoting the density part of $\sum_{i=1}^{N(t)} Y_{i}-c t$ at $y$ by $g(y, t)$ for $-\infty<y<\infty$ and $t>0$, we have

$$
\mathbb{E}\left[e^{-s\left(\sum_{i=1}^{N(t)} Y_{i}-c t\right)}\right]=\int_{-\infty}^{\infty} e^{-s y} g(y, t) d y+e^{-s(-c t)} e^{-\lambda t},
$$

and it will be convenient to write

$$
g(y, t)=g_{-}(-y, t) I_{\{y<0\}}+g_{+}(y, t) I_{\{y>0\}}, \quad-\infty<y<\infty, t>0 .
$$

Clearly $g(y, t)=0$ for $y \leq-c t$ so that $g_{-}(y, t)=0$ for $y>c t$. By the tower property and a change of variables this leads to

$$
\begin{aligned}
\mathbb{E}\left[e^{-\delta T-s\left(\sum_{i=1}^{N(T)} Y_{i}-c T\right)}\right] & =\mathbb{E}\left[e^{-\delta T} \mathbb{E}\left[e^{-s\left(\sum_{i=1}^{N(T)} Y_{i}-c T\right)} \mid T\right]\right] \\
& =\int_{0}^{\infty} e^{-\delta t}\left[\int_{-\infty}^{\infty} e^{-s y} g(y, t) d y+e^{-s(-c t)} e^{-\lambda t}\right] \frac{\gamma^{n} t^{n-1} e^{-\gamma t}}{(n-1) !} d t \\
& =\int_{-\infty}^{\infty} e^{-s y} \int_{0}^{\infty} \frac{\gamma^{n} t^{n-1} e^{-(\gamma+\delta) t}}{(n-1) !} g(y, t) d t d y+\frac{1}{c} \int_{-\infty}^{0} e^{-s y} \frac{\gamma^{n}\left(-\frac{y}{c}\right)^{n-1} e^{\frac{\lambda+\gamma+\delta}{c} y}}{(n-1) !} d y
\end{aligned}
$$


so that a comparison with (3.3) gives

$$
g_{\delta}(y)=\int_{0}^{\infty} \frac{\gamma^{n} t^{n-1} e^{-(\gamma+\delta) t}}{(n-1) !} g(y, t) d t+\frac{1}{c} \frac{\gamma^{n}\left(-\frac{y}{c}\right)^{n-1} e^{\frac{\lambda+\gamma+\delta}{c} y}}{(n-1) !} I_{\{y<0\}}, \quad-\infty<y<\infty .
$$

Due to (3.4) and (3.5), one concludes that $g_{\delta,-}(\cdot)$ and $g_{\delta,+}(\cdot)$ are given by

$$
g_{\delta,-}(y)=\int_{0}^{\frac{y}{c}} \frac{\gamma^{n} t^{n-1} e^{-(\gamma+\delta) t}}{(n-1) !} g_{-}(y, t) d t+\frac{1}{c} \frac{\gamma^{n}\left(\frac{y}{c}\right)^{n-1} e^{-\frac{\lambda+\gamma+\delta}{c} y}}{(n-1) !}, \quad y>0,
$$

and

$$
g_{\delta,+}(y)=\int_{0}^{\infty} \frac{\gamma^{n} t^{n-1} e^{-(\gamma+\delta) t}}{(n-1) !} g_{+}(y, t) d t, \quad y>0
$$

respectively. Although the definitions (3.6) and (3.7) via $g(y, t)$ will not be used in our subsequent analysis, they may be helpful to clarify the meaning of the discounted densities $g_{\delta,-}(\cdot)$ and $g_{\delta,+}(\cdot)$.

The denominator in the bracket on the right-hand side of the double-sided Laplace transform (3.2) of $g_{\delta}(y)$ is in the form of a Lundberg-type equation (in $\xi$ )

$$
c \xi-(\lambda+\gamma+\delta)+\lambda \widetilde{f}_{Y}(\xi)=0
$$

which, due to $\gamma+\delta>0$, has a unique positive root $\rho_{\gamma}>0$ (note that equation (2.8) was the special case of exponential claims). To make the following analysis more transparent, we will use the Dickson-Hipp operator $\mathcal{T}_{s}$ (see e.g. Dickson \& Hipp [8]), which for any integrable function $f(\cdot)$ on $(0, \infty)$ and any complex number $s$ with $\operatorname{Re}(s) \geq 0$ is defined as

$$
\mathcal{T}_{s} f(y)=\int_{y}^{\infty} e^{-s(z-y)} f(z) d z=\int_{0}^{\infty} e^{-s z} f(z+y) d z, \quad y \geq 0 .
$$

Equation (3.2) can then be expressed as

$$
\begin{aligned}
\mathbb{E}\left[e^{-\delta T-s\left(\sum_{i=1}^{N(T)} Y_{i}-c T\right)}\right] & =\left(\frac{\gamma}{c\left(\rho_{\gamma}-s\right)-\lambda\left[\widetilde{f}_{Y}(s)-\widetilde{f}_{Y}\left(\rho_{\gamma}\right)\right]}\right)^{n}=\left(\frac{\gamma}{c \rho_{\gamma}} \frac{\rho_{\gamma}}{\rho_{\gamma}-s} \frac{1}{1-\frac{\lambda}{c} \frac{\widetilde{f}_{Y}(s)-\widetilde{f}_{Y}\left(\rho_{\gamma}\right)}{\rho_{\gamma}-s}}\right)^{n} \\
& =\left(\frac{\gamma}{c \rho_{\gamma}} \frac{\rho_{\gamma}}{\rho_{\gamma}-s} \frac{1}{1-\phi \widetilde{f}_{L}(s)}\right)^{n}=\left(\frac{\gamma}{\gamma+\delta}\right)^{n}\left(\frac{\rho_{\gamma}}{\rho_{\gamma}-s}\right)^{n}\left(\frac{1-\phi}{1-\phi \widetilde{f}_{L}(s)}\right)^{n}
\end{aligned}
$$

where

$$
\phi=\frac{\lambda}{c} \mathcal{T}_{0} \mathcal{T}_{\rho_{\gamma}} f_{Y}(0)=1-\frac{\gamma+\delta}{c \rho_{\gamma}}<1
$$

and

$$
\widetilde{f}_{L}(s)=\frac{\mathcal{T}_{s} \mathcal{T}_{\rho_{\gamma}} f_{Y}(0)}{\mathcal{T}_{0} \mathcal{T}_{\rho_{\gamma}} f_{Y}(0)}
$$

is the Laplace transform of the proper density

$$
f_{L}(y)=\frac{\mathcal{T}_{\rho_{\gamma}} f_{Y}(y)}{\mathcal{T}_{0} \mathcal{T}_{\rho_{\gamma}} f_{Y}(0)}, \quad y>0
$$


which corresponds to a generic r.v. L. Here we have used the fact that the Dickson-Hipp operator satisfies

$$
\mathcal{T}_{s_{1}} \mathcal{T}_{s_{2}} f(y)=\mathcal{T}_{s_{2}} \mathcal{T}_{s_{1}} f(y)=\frac{\mathcal{T}_{s_{1}} f(y)-\mathcal{T}_{s_{2}} f(y)}{s_{2}-s_{1}}, \quad y \geq 0
$$

for all complex numbers $s_{1} \neq s_{2}$ (see e.g. Li \& Garrido [12, Sec. 3]).

It is instructive to note that in the expression (3.9), $\left[\rho_{\gamma} /\left(\rho_{\gamma}-s\right)\right]^{n}$ is the Laplace transform (with $\operatorname{argument}-s)$ of an $\operatorname{Erlang}(n)$ r.v. with density $\rho_{\gamma}^{n} y^{n-1} e^{-\rho_{\gamma} y} /(n-1)$ !, whereas $\left[(1-\phi) /\left(1-\phi \widetilde{f}_{L}(s)\right)\right]^{n}$ is the Laplace transform (with argument $s$ ) of a compound negative binomial r.v. with primary probability mass function at $k(k=0,1, \ldots)$ being $\left(\begin{array}{c}n+k-1 \\ n-1\end{array}\right) \phi^{k}(1-\phi)^{n}$ and secondary density $f_{L}(\cdot)$. Since $[\gamma /(\gamma+\delta)]^{n} \leq 1$, comparison of (3.3) and (3.9) reveals that $g_{\delta}(y)$ is the density of the compound negative binomial r.v. minus an independent $\operatorname{Erlang}(n)$ r.v., which is defective when $\delta>0$. Hence $g_{\delta,-}(\cdot)$ represents the density when the Erlang r.v. is larger while $g_{\delta,+}(\cdot)$ is the case where the compound negative binomial r.v. is larger. Using this observation, we first consider $g_{\delta,-}(\cdot)$. Noting that the above compound binomial r.v. has a point mass at 0 , we arrive at

$$
\begin{aligned}
g_{\delta,-}(y) & =\left(\frac{\gamma}{\gamma+\delta}\right)^{n}\left[(1-\phi)^{n} \frac{\rho_{\gamma}^{n} y^{n-1} e^{-\rho_{\gamma} y}}{(n-1) !}+\int_{y}^{\infty} \frac{\rho_{\gamma}^{n} z^{n-1} e^{-\rho_{\gamma} z}}{(n-1) !} \sum_{k=1}^{\infty}\left(\begin{array}{c}
n+k-1 \\
n-1
\end{array}\right) \phi^{k}(1-\phi)^{n} f_{L}^{* k}(z-y) d z\right] \\
& =\left(\frac{\gamma}{c}\right)^{n}\left[\frac{y^{n-1} e^{-\rho_{\gamma} y}}{(n-1) !}+\sum_{k=1}^{\infty}\left(\begin{array}{c}
n+k-1 \\
n-1
\end{array}\right) \phi^{k} \int_{y}^{\infty} \frac{z^{n-1} e^{-\rho_{\gamma} z}}{(n-1) !} f_{L}^{* k}(z-y) d z\right], \quad y>0, \quad
\end{aligned}
$$

where the last equality follows from (3.10). Here $f_{L}^{* k}(\cdot)$ denotes the $k$-fold convolution of $f_{L}(\cdot)$ with itself defined recursively via $f_{L}^{* k}(y)=\int_{0}^{y} f_{L}^{* k-1}(y-z) f_{L}(z) d z$ for $k \geq 2$ and $f_{L}^{* 1}(\cdot)=f_{L}(\cdot)$. The integral term above can further be written as

$$
\begin{aligned}
\int_{y}^{\infty} \frac{z^{n-1} e^{-\rho_{\gamma} z}}{(n-1) !} f_{L}^{* k}(z-y) d z & =\frac{1}{(n-1) !} \int_{0}^{\infty}(z+y)^{n-1} e^{-\rho_{\gamma}(z+y)} f_{L}^{* k}(z) d z \\
& =\frac{1}{(n-1) !} \sum_{j=1}^{n}\left(\begin{array}{c}
n-1 \\
j-1
\end{array}\right) y^{j-1} e^{-\rho_{\gamma} y} \int_{0}^{\infty} z^{n-j} e^{-\rho_{\gamma} z} f_{L}^{* k}(z) d z \\
& =\sum_{j=1}^{n} \frac{y^{j-1} e^{-\rho_{\gamma} y}}{(j-1) !} \int_{0}^{\infty} \frac{z^{n-j} e^{-\rho_{\gamma} z}}{(n-j) !} f_{L}^{* k}(z) d z \\
& =\sum_{j=1}^{n} \frac{y^{j-1} e^{-\rho_{\gamma} y}}{(j-1) !} \mathcal{T}_{\rho_{\gamma}-j+1}^{n} f_{L}^{* k}(0),
\end{aligned}
$$

where $\mathcal{T}_{s}^{j}$ stands for the multiple Dickson-Hipp operator of order $j$ (see e.g. Li \& Garrido [12, Section 3, Property 5]). Substitution of (3.14) into (3.13) leads to

$$
g_{\delta,-}(y)=\sum_{j=1}^{n} B_{j}^{*} \frac{y^{j-1} e^{-\rho_{\gamma} y}}{(j-1) !}, \quad y>0
$$

with

$$
B_{j}^{*}=\left(\frac{\gamma}{c}\right)^{n} \sum_{k=1}^{\infty}\left(\begin{array}{c}
n+k-1 \\
n-1
\end{array}\right) \phi^{k} \mathcal{T}_{\rho_{\gamma}}^{n-j+1} f_{L}^{* k}(0), \quad j=1,2, \ldots, n-1
$$


and

$$
\begin{aligned}
B_{n}^{*} & =\left(\frac{\gamma}{c}\right)^{n}\left[1+\sum_{k=1}^{\infty}\left(\begin{array}{c}
n+k-1 \\
n-1
\end{array}\right) \phi^{k} \mathcal{T}_{\rho_{\gamma}} f_{L}^{* k}(0)\right]=\left(\frac{\gamma}{c}\right)^{n} \sum_{k=0}^{\infty}\left(\begin{array}{c}
n+k-1 \\
n-1
\end{array}\right) \phi^{k}\left[\widetilde{f}_{L}\left(\rho_{\gamma}\right)\right]^{k} \\
& =\left(\frac{\gamma}{c}\right)^{n} \frac{1}{\left[1-\phi \widetilde{f}_{L}\left(\rho_{\gamma}\right)\right]^{n}} .
\end{aligned}
$$

Note that (3.15) implies that $g_{\delta,-}(y)$ is a (defective) mixture of Erlang distributions.

Similarly, one arrives at

$$
\begin{aligned}
g_{\delta,+}(y) & =\left(\frac{\gamma}{\gamma+\delta}\right)^{n} \int_{y}^{\infty} \sum_{k=1}^{\infty}\left(\begin{array}{c}
n+k-1 \\
n-1
\end{array}\right) \phi^{k}(1-\phi)^{n} f_{L}^{* k}(z) \frac{\rho_{\gamma}^{n}(z-y)^{n-1} e^{-\rho_{\gamma}(z-y)}}{(n-1) !} d z \\
& =\left(\frac{\gamma}{c}\right)^{n} \sum_{k=1}^{\infty}\left(\begin{array}{c}
n+k-1 \\
n-1
\end{array}\right) \phi^{k} \mathcal{T}_{\rho_{\gamma}}^{n} f_{L}^{* k}(y), \quad y>0 .
\end{aligned}
$$

\subsection{The discounted densities $h_{1, \delta}^{*}(y \mid 0)$ and $h_{2, \delta}^{*}(z, y \mid 0)$}

Recall from Section 3.1 that our goal is to identify the discounted densities $h_{1, \delta}^{*}(\cdot \mid 0)$ and $h_{2, \delta}^{*}(\cdot, \cdot \mid 0)$. By definition,

$$
h_{1, \delta}^{*}(y \mid 0)=g_{\delta,+}(y), \quad y>0 .
$$

As we shall see in this subsection, $h_{2, \delta}^{*}(\cdot, \cdot \mid 0)$ can also be expressed in terms of $g_{\delta,+}(\cdot)$, with $g_{\delta,-}(\cdot)$ given in (3.15) playing a crucial role.

Conditioning on the increment $\sum_{i=1}^{N(T)} Y_{i}-c T$, one arrives at the integral equation

$$
m_{\delta}(x)=\int_{0}^{\infty} m_{\delta}(x+y) g_{\delta,-}(y) d y+\int_{0}^{x} m_{\delta}(x-y) g_{\delta,+}(y) d y+\int_{x}^{\infty} w(x, y-x) g_{\delta,+}(y) d y, \quad x \geq 0 .
$$

W.l.o.g. we choose the penalty function $w\left(x_{1}, x_{2}\right)=e^{-r_{1} x_{1}-r_{2} x_{2}}$. Substituting the form of (3.15), (3.20) becomes

$$
\begin{aligned}
m_{\delta}(x) & =\int_{0}^{\infty} \sum_{j=1}^{n} B_{j}^{*} \frac{y^{j-1} e^{-\rho_{\gamma} y}}{(j-1) !} m_{\delta}(x+y) d y+\int_{0}^{x} m_{\delta}(x-y) g_{\delta,+}(y) d y+\int_{x}^{\infty} e^{-r_{1} x-r_{2}(y-x)} g_{\delta,+}(y) d y \\
& =\sum_{j=1}^{n} B_{j}^{*} \mathcal{T}_{\rho_{\gamma}}^{j} m_{\delta}(x)+\int_{0}^{x} m_{\delta}(x-y) g_{\delta,+}(y) d y+\varphi_{\delta}(x), \quad x \geq 0
\end{aligned}
$$

where

$$
\varphi_{\delta}(x)=\int_{0}^{\infty} e^{-r_{1} x-r_{2} y} g_{\delta,+}(x+y) d y, \quad x \geq 0 .
$$

Again using Property 5 of Li \& Garrido [12, Sec. 3] regarding the Laplace transform of multiple DicksonHipp operators, taking Laplace transforms on both sides of (3.21) results in

$$
\widetilde{m}_{\delta}(s)=\sum_{j=1}^{n} B_{j}^{*}\left(\frac{\widetilde{m}_{\delta}(s)}{\left(\rho_{\gamma}-s\right)^{j}}-\sum_{l=1}^{j} \frac{\mathcal{T}_{\rho_{\gamma}}^{l} m_{\delta}(0)}{\left(\rho_{\gamma}-s\right)^{j+1-l}}\right)+\widetilde{g}_{\delta,+}(s) \widetilde{m}_{\delta}(s)+\widetilde{\varphi}_{\delta}(s),
$$


where $\widetilde{m}_{\delta}(s)=\int_{0}^{\infty} e^{-s x} m_{\delta}(x) d x, \widetilde{g}_{\delta,+}(s)=\int_{0}^{\infty} e^{-s y} g_{\delta,+}(y) d y$, and

$$
\widetilde{\varphi}_{\delta}(s)=\int_{0}^{\infty} e^{-s x} \varphi_{\delta}(x) d x=\int_{0}^{\infty} \int_{0}^{\infty} e^{-r_{1} x-r_{2} y} e^{-s x} g_{\delta,+}(x+y) d x d y .
$$

With (3.15), (3.23) can also be written as

$$
\left[1-\widetilde{g}_{\delta,-}(-s)-\widetilde{g}_{\delta,+}(s)\right] \widetilde{m}_{\delta}(s)=\widetilde{\varphi}_{\delta}(s)-\frac{1}{\left(\rho_{\gamma}-s\right)^{n}} P_{\delta}(s)
$$

where

$$
\widetilde{g}_{\delta,-}(-s)=\int_{0}^{\infty} e^{s y} g_{\delta,-}(y) d y=\int_{-\infty}^{0} e^{-s y} g_{\delta,-}(-y) d y
$$

and

$$
P_{\delta}(s)=\sum_{j=1}^{n} B_{j}^{*} \sum_{l=1}^{j} \mathcal{T}_{\rho_{\gamma}}^{l} m_{\delta}(0)\left(\rho_{\gamma}-s\right)^{n-(j+1-l)}
$$

is a polynomial (in $s$ ) of degree (at most) $n-1$. Thus, application of (3.3) gives

$$
\widetilde{m}_{\delta}(s)=\frac{\widetilde{\varphi}_{\delta}(s)-P_{\delta}(s) /\left(\rho_{\gamma}-s\right)^{n}}{1-\mathbb{E}\left[e^{-\delta T-s\left(\sum_{i=1}^{N(T)} Y_{i}-c T\right)}\right]}
$$

The denominator of this ratio is the (generalized) Lundberg fundamental equation (in $\xi$ )

$$
\mathbb{E}\left[e^{-\delta T-\xi\left(\sum_{i=1}^{N(T)} Y_{i}-c T\right)}\right]=1,
$$

in accordance with the associated dependent Sparre Andersen risk model. In Appendix A it is shown that this equation has exactly $n$ roots $\left\{\alpha_{i}\right\}_{i=1}^{n}$ with non-negative real part (under $\delta>0$ or the positive loading condition $c>\lambda \mathbb{E}[Y]$ ). In what follows, we will assume these $n$ roots to be distinct.

For $r_{1}, r_{2} \geq 0, \widetilde{m}_{\delta}(s)$ is a bounded analytic function for $\operatorname{Re}(s) \geq 0$, so these $n$ zeros of the denominator also need to be zeros of the numerator. The Lagrange interpolation formula and the Initial Value Theorem for Laplace transforms then leads to

$$
m_{\delta}(0)=\varphi_{\delta}(0)+\sum_{i=1}^{n} \vartheta_{i} \widetilde{\varphi}_{\delta}\left(\alpha_{i}\right)
$$

where

$$
\vartheta_{i}=\frac{\left(\rho_{\gamma}-\alpha_{i}\right)^{n}}{\prod_{j=1, j \neq i}^{n}\left(\alpha_{j}-\alpha_{i}\right)}, \quad i=1,2, \ldots, n .
$$

Substituting (3.22) and (3.24) into (3.26) now yields

$$
m_{\delta}(0)=\int_{0}^{\infty} e^{-r_{2} y} g_{\delta,+}(y) d y+\sum_{i=1}^{n} \vartheta_{i} \int_{0}^{\infty} \int_{0}^{\infty} e^{-r_{1} z-r_{2} y} e^{-\alpha_{i} z} g_{\delta,+}(z+y) d z d y
$$

Finally, a comparison of this equation with (3.1) for $x=0$ and $w\left(x_{1}, x_{2}\right)=e^{-r_{1} x_{1}-r_{2} x_{2}}$ establishes

$$
h_{2, \delta}^{*}(z, y \mid 0)=\sum_{i=1}^{n} \vartheta_{i} e^{-\alpha_{i} z} g_{\delta,+}(z+y), \quad z, y>0 .
$$

Note that the form of (3.27) resembles Equation (16) of Li \& Garrido [13] for the classical Sparre Andersen risk model with Coxian interclaim time distribution. 
Remark 3.2 For $n=1$, it is shown in Appendix B that

$$
\lim _{\gamma \rightarrow \infty} h_{1, \delta}^{*}(y \mid 0)=0 \quad \text { for almost all } y>0,
$$

and

$$
\lim _{\gamma \rightarrow \infty} h_{2, \delta}^{*}(z, y \mid 0)=\frac{\lambda}{c} e^{-\rho_{0} z} f_{Y}(z+y) \quad \text { for almost all } z, y>0 .
$$

Equation (3.29) is indeed the discounted joint density of the surplus prior to ruin and the deficit at ruin in the classical compound Poisson risk model for $x=0$ (cf. Gerber \& Shiu [10, Eqn. (3.3)]).

Remark 3.3 If time 0 is not an observation time (and hence $x \in \mathbb{R}$ ), and the time until the first observation is $\operatorname{Erlang}(n)$ distributed, then $m_{\delta, U}(x)(x \geq 0)$ is just the $m_{\delta}(x)$ studied above, and $m_{\delta, L}(x)$ for $x<0$ can be expressed in terms of $m_{\delta, U}(\cdot)$ and the discounted densities $g_{\delta,-}(\cdot)$ and $g_{\delta,+}(\cdot)$ via

$m_{\delta, L}(x)=\int_{-x}^{\infty} m_{\delta, U}(x+y) g_{\delta,-}(y) d y+\int_{0}^{-x} w(x,-y-x) g_{\delta,-}(y) d y+\int_{0}^{\infty} w(x, y-x) g_{\delta,+}(y) d y, \quad x<0$.

\section{Analysis for claims with rational Laplace transform}

In Section 3, we showed that $m_{\delta}(x)$ is the solution of a defective renewal equation that involves, via the discounted densities $h_{1, \delta}^{*}(\cdot \mid 0)$ and $h_{2, \delta}^{*}(\cdot, \cdot \mid 0)$, the function $g_{\delta,+}(\cdot)$, which in turn is given by $(3.18)$. However, (3.18) involves (the infinite sum of) multiple Dickson-Hipp operators applied to $k$-fold convolutions, which in general does not admit closed-form representations. In this section, we will illustrate that for claim sizes $Y$ with rational Laplace transform, more tractable expressions for $g_{\delta,+}($.$) and g_{\delta,-}($.$) , and subsequently$ for $m_{\delta}(x)$, can be obtained.

Suppose therefore in the remainder of this section that

$$
\widetilde{f}_{Y}(s)=\frac{Q_{2, r-1}(s)}{Q_{1, r}(s)}
$$

where $Q_{1, r}(s)$ is a polynomial in $s$ of degree $r$ and $Q_{2, r-1}(s)$ is a polynomial in $s$ of degree at most $r-1$. W.l.o.g. suppose that $Q_{1, r}(s)$ and $Q_{2, r-1}(s)$ have no common zeros, and $Q_{1, r}(s)$ has leading coefficient 1.

\subsection{The discounted densities $g_{\delta,-}(y)$ and $g_{\delta,+}(y)$}

Applying property (3.12) to (3.11), one can show that $\widetilde{f}_{L}(s)=Q_{2, r-1}^{*}(s) / Q_{1, r}(s)$ where $Q_{2, r-1}^{*}(s)$ is again a polynomial in $s$ of degree at most $r-1$. Denote with $\left\{-\kappa_{i}\right\}_{i=1}^{r}$ the $r$ roots of the polynomial equation $\widetilde{f}_{L}(\xi)=1 / \phi($ in $\xi)$, i.e. of

$$
Q_{1, r}(\xi)-\phi Q_{2, r-1}^{*}(\xi)=0
$$


and assume for simplicity that all these roots are distinct. Since $\phi<1$, all these roots must have negative real parts and are in fact also the roots with negative real parts of the Lundberg-type equation (3.8). Using (3.10), (3.9) becomes

$$
\mathbb{E}\left[e^{-\delta T-s\left(\sum_{i=1}^{N(T)} Y_{i}-c T\right)}\right]=\left(\frac{\gamma}{\gamma+\delta}\right)^{n}\left(\frac{\rho_{\gamma}}{\rho_{\gamma}-s}\right)^{n}\left(\frac{(1-\phi) Q_{1, r}(s)}{\prod_{i=1}^{r}\left(s+\kappa_{i}\right)}\right)^{n}=\left(\frac{\gamma}{c}\right)^{n} \frac{\left[Q_{1, r}(s)\right]^{n}}{\left(\rho_{\gamma}-s\right)^{n} \prod_{i=1}^{r}\left(s+\kappa_{i}\right)^{n}},
$$

which by partial fractions translates into

$$
\mathbb{E}\left[e^{-\delta T-s\left(\sum_{i=1}^{N(T)} Y_{i}-c T\right)}\right]=\sum_{j=1}^{n} \frac{B_{j}^{*}}{\left(\rho_{\gamma}-s\right)^{j}}+\sum_{i=1}^{r} \sum_{j=1}^{n} \frac{B_{i j}}{\left(s+\kappa_{i}\right)^{j}},
$$

where

$$
B_{j}^{*}=\left.(-1)^{n-j}\left(\frac{\gamma}{c}\right)^{n} \frac{1}{(n-j) !} \frac{d^{n-j}}{d s^{n-j}} \frac{\left[Q_{1, r}(s)\right]^{n}}{\prod_{l=1}^{r}\left(s+\kappa_{l}\right)^{n}}\right|_{s=\rho_{\gamma}}, \quad j=1,2, \ldots, n,
$$

and

$$
B_{i j}=\left.\left(\frac{\gamma}{c}\right)^{n} \frac{1}{(n-j) !} \frac{d^{n-j}}{d s^{n-j}} \frac{\left[Q_{1, r}(s)\right]^{n}}{\left(\rho_{\gamma}-s\right)^{n} \prod_{l=1, l \neq i}^{r}\left(s+\kappa_{l}\right)^{n}}\right|_{s=-\kappa_{i}}, \quad i=1,2, \ldots, r ; j=1,2, \ldots, n .
$$

Combining (3.3) and (4.1) shows that $g_{\delta}(y)$ is of the form (3.4) with $g_{\delta,-}(y)$ given by (3.15) and

$$
g_{\delta,+}(y)=\sum_{i=1}^{r} \sum_{j=1}^{n} B_{i j} \frac{y^{j-1} e^{-\kappa_{i} y}}{(j-1) !}, \quad y>0 .
$$

We remark that the $B_{j}^{*}$ 's given in (4.2) are identical to those given by (3.16) and (3.17). However, from a computational point of view, it is more convenient to apply (4.2). With regards to $g_{\delta,+}(y),(4.3)$ is also more tractable than (3.18). Indeed, apart from a constant multiple, the function $g_{\delta,+}(y)$ corresponds to a combination of Erlangs when all $\left\{\kappa_{i}\right\}_{i=1}^{r}$ are real (which is for instance the case when $Y$ is a mixture of exponentials).

Example 4.1 For the model considered in Section 2 in which the claim size $Y$ and the observation time $T$ are both exponentially distributed with mean $1 / \nu$ and $1 / \gamma$, respectively, we have $n=1$ and $\kappa_{1}=R_{\gamma}$. The partial fractions technique above then leads to

$$
g_{\delta}(y)=\frac{\gamma\left(\nu+\rho_{\gamma}\right)}{c \rho_{\gamma}\left(\rho_{\gamma}+R_{\gamma}\right)} \rho_{\gamma} e^{\rho_{\gamma} y} I_{\{y<0\}}+\frac{\gamma\left(\nu-R_{\gamma}\right)}{c R_{\gamma}\left(\rho_{\gamma}+R_{\gamma}\right)} R_{\gamma} e^{-R_{\gamma} y} I_{\{y>0\}}, \quad-\infty<y<\infty,
$$

which is a (defective) two-sided exponential density. Consequently, from (3.27),

$$
h_{2, \delta}^{*}(z, y \mid 0)=\frac{\gamma\left(\nu-R_{\gamma}\right)\left(\rho_{\gamma}-\rho_{0}\right)}{c\left(\rho_{\gamma}+R_{\gamma}\right)} e^{-\left(R_{\gamma}+\rho_{0}\right) z-R_{\gamma} y}, \quad z, y>0 .
$$

Suppose now $w\left(x_{1}, x_{2}\right)=e^{-r_{1} x_{1}} w_{2}\left(x_{2}\right)$ (see e.g. Willmot (2007)). Then, with (2.17) at $x=0$ and some simple manipulations, the defective renewal equation (3.1) reads

$$
\begin{aligned}
m_{\delta}(x)= & \int_{0}^{x} m_{\delta}(x-y)\left(R_{\gamma}-R_{0}\right) e^{-R_{\gamma} y} d y+\int_{x}^{\infty} e^{-r_{1} x} w_{2}(y-x) \frac{\gamma\left(\nu-R_{\gamma}\right)}{c\left(\rho_{\gamma}+R_{\gamma}\right)} e^{-R_{\gamma} y} d y \\
& +\int_{x}^{\infty} \int_{0}^{\infty} e^{-r_{1}(z+x)} w_{2}(y-x) \frac{\gamma\left(\nu-R_{\gamma}\right)\left(\rho_{\gamma}-\rho_{0}\right)}{c\left(\rho_{\gamma}+R_{\gamma}\right)} e^{-\left(R_{\gamma}+\rho_{0}\right) z-R_{\gamma} y} d z d y \\
= & \left(R_{\gamma}-R_{0}\right) \int_{0}^{x} m_{\delta}(x-y) e^{-R_{\gamma} y} d y \\
& +\frac{\gamma\left(\nu-R_{\gamma}\right)}{c\left(\rho_{\gamma}+R_{\gamma}\right)} \frac{r_{1}+\rho_{\gamma}+R_{\gamma}}{r_{1}+R_{\gamma}+\rho_{0}}\left(\int_{0}^{\infty} w_{2}(y) e^{-R_{\gamma} y} d y\right) e^{-\left(r_{1}+R_{\gamma}\right) x}, \quad x \geq 0 .
\end{aligned}
$$


Taking Laplace transform with respect to $x$ and then solving for $\widetilde{m}_{\delta}(s)$ yields

$$
\begin{aligned}
\widetilde{m}_{\delta}(s) & =\frac{\gamma\left(\nu-R_{\gamma}\right)}{c\left(\rho_{\gamma}+R_{\gamma}\right)} \frac{r_{1}+\rho_{\gamma}+R_{\gamma}}{r_{1}+R_{\gamma}+\rho_{0}}\left(\int_{0}^{\infty} w_{2}(y) e^{-R_{\gamma} y} d y\right) \frac{s+R_{\gamma}}{\left(s+R_{0}\right)\left(s+r_{1}+R_{\gamma}\right)} \\
& =\frac{\gamma\left(\nu-R_{\gamma}\right)}{c\left(\rho_{\gamma}+R_{\gamma}\right)} \frac{r_{1}+\rho_{\gamma}+R_{\gamma}}{\left(r_{1}+R_{\gamma}+\rho_{0}\right)\left(r_{1}+R_{\gamma}-R_{0}\right)}\left(\int_{0}^{\infty} w_{2}(y) e^{-R_{\gamma} y} d y\right)\left(\frac{R_{\gamma}-R_{0}}{s+R_{0}}+\frac{r_{1}}{s+r_{1}+R_{\gamma}}\right) .
\end{aligned}
$$

But this expression can be explicitly inverted to give

$$
\begin{aligned}
m_{\delta}(x)=\frac{\gamma\left(\nu-R_{\gamma}\right)}{c\left(\rho_{\gamma}+R_{\gamma}\right)} \frac{r_{1}+\rho_{\gamma}+R_{\gamma}}{\left(r_{1}+R_{\gamma}+\rho_{0}\right)\left(r_{1}+R_{\gamma}-R_{0}\right)} & \\
& \cdot\left(\int_{0}^{\infty} w_{2}(y) e^{-R_{\gamma} y} d y\right)\left[\left(R_{\gamma}-R_{0}\right) e^{-R_{0} x}+r_{1} e^{-\left(r_{1}+R_{\gamma}\right) x}\right], \quad x \geq 0 .
\end{aligned}
$$

So in this case we have demonstrated that the defective renewal equation (3.1) can be solved directly via Laplace transform and inversion. In principle, the arguments also extend to claims with rational Laplace transform using the expression (4.3), the corresponding analysis is however tedious.

\subsection{Explicit expression for $m_{\delta}(x)$ when $w\left(x_{1}, x_{2}\right) \equiv w_{2}\left(x_{2}\right)$}

Suppose now that the penalty function depends on the deficit only, i.e. $w\left(x_{1}, x_{2}\right) \equiv w_{2}\left(x_{2}\right)$. Then $(3.20)$ simplifies to

$$
m_{\delta}(x)=\int_{0}^{\infty} m_{\delta}(x+y) g_{\delta,-}(y) d y+\int_{0}^{x} m_{\delta}(x-y) g_{\delta,+}(y) d y+\int_{x}^{\infty} w_{2}(y-x) g_{\delta,+}(y) d y, \quad x \geq 0 .
$$

For claims with rational Laplace transform, one can indeed directly solve (4.5) for $m_{\delta}(x)$ due to the nice form of the density $g_{\delta,+}(y)$ given in (4.3), and this is done in what follows.

Applying the operator $\left(d / d x-\rho_{\gamma}\right)^{n} \prod_{i=1}^{r}\left(d / d x+\kappa_{i}\right)^{n}$ to both sides of (4.5), we observe that $m_{\delta}(x)$ satisfies a homogeneous differential equation in $x$ of order $n(r+1)$ with constant coefficients. Therefore, the solution is of the form

$$
m_{\delta}(x)=\sum_{i=1}^{n(r+1)} C_{i} e^{\alpha_{i} x}, \quad x \geq 0,
$$

where $\left\{C_{i}\right\}_{i=1}^{n(r+1)}$ and $\left\{\alpha_{i}\right\}_{i=1}^{n(r+1)}$ are constants to be determined. Assuming that $\lim _{x \rightarrow \infty} m_{\delta}(x)=0$, we have that $C_{j}=0$ whenever $\alpha_{j} \geq 0$ for any $j=1,2, \ldots, n(r+1)$. Our next step involves direct substitution of (3.15), (4.3) and (4.6) into (4.5) to determine the unknown constants. The first integral on the right-hand side of (4.5) is then given by

$$
\begin{aligned}
& \int_{0}^{\infty} m_{\delta}(x+y) g_{\delta,-}(y) d y=\int_{0}^{\infty} \sum_{i=1}^{n(r+1)} C_{i} e^{\alpha_{i}(x+y)} \sum_{j=1}^{n} B_{j}^{*} \frac{y^{j-1} e^{-\rho_{\gamma} y}}{(j-1) !} d y \\
= & \sum_{i=1}^{n(r+1)} C_{i} \sum_{j=1}^{n} B_{j}^{*}\left(\int_{0}^{\infty} \frac{y^{j-1} e^{-\left(\rho_{\gamma}-\alpha_{i}\right) y}}{(j-1) !} d y\right) e^{\alpha_{i} x}=\sum_{i=1}^{n(r+1)} C_{i}\left(\sum_{j=1}^{n} \frac{B_{j}^{*}}{\left(\rho_{\gamma}-\alpha_{i}\right)^{j}}\right) e^{\alpha_{i} x} .
\end{aligned}
$$


We remark that the integral in the second equality only exists when $\rho_{\gamma}>\alpha_{i}$ for all $i=1,2, \ldots, n(r+1)$. However, if $\alpha_{j} \geq \rho_{\gamma}$ for some $j$, then the corresponding $C_{j}$ is 0 , and therefore the above equation always holds true. Next, the second integral in (4.5) is evaluated as

$$
\begin{aligned}
& \int_{0}^{x} m_{\delta}(x-y) g_{\delta,+}(y) d y=\int_{0}^{x} \sum_{i=1}^{n(r+1)} C_{i} e^{\alpha_{i}(x-y)} \sum_{k=1}^{r} \sum_{j=1}^{n} B_{k j} \frac{y^{j-1} e^{-\kappa_{k} y}}{(j-1) !} d y \\
= & \sum_{i=1}^{n(r+1)} C_{i} \sum_{k=1}^{r} \sum_{j=1}^{n} B_{k j}\left(\int_{0}^{x} \frac{y^{j-1} e^{-\left(\kappa_{k}+\alpha_{i}\right) y}}{(j-1) !} d y\right) e^{\alpha_{i} x} \\
= & \sum_{i=1}^{n(r+1)} C_{i} \sum_{k=1}^{r} \sum_{j=1}^{n} \frac{B_{k j}}{\left(\kappa_{k}+\alpha_{i}\right)^{j}}\left(1-\sum_{z=0}^{j-1} e^{-\left(\kappa_{k}+\alpha_{i}\right) x} \frac{\left[\left(\kappa_{k}+\alpha_{i}\right) x\right]^{z}}{z !}\right) e^{\alpha_{i} x} \\
= & \sum_{i=1}^{n(r+1)} C_{i}\left(\sum_{k=1}^{r} \sum_{j=1}^{n} \frac{B_{k j}}{\left(\kappa_{k}+\alpha_{i}\right)^{j}}\right) e^{\alpha_{i} x}-\sum_{k=1}^{r} \sum_{i=1}^{n}\left(\sum_{z=1}^{n(r+1)} C_{z} \sum_{j=i}^{n} \frac{B_{k j}}{\left(\kappa_{k}+\alpha_{z}\right)^{j-i+1}}\right) \frac{x^{i-1} e^{-\kappa_{k} x}}{(i-1) !} .
\end{aligned}
$$

Finally, the third integral in (4.5) is given by

$$
\begin{aligned}
& \int_{x}^{\infty} w_{2}(y-x) g_{\delta,+}(y) d y=\int_{0}^{\infty} w_{2}(y) \sum_{k=1}^{r} \sum_{j=1}^{n} B_{k j} \frac{(y+x)^{j-1} e^{-\kappa_{k}(y+x)}}{(j-1) !} d y \\
= & \sum_{k=1}^{r} \sum_{j=1}^{n} \frac{B_{k j}}{(j-1) !} \sum_{i=1}^{j}\left(\begin{array}{c}
j-1 \\
i-1
\end{array}\right)\left(\int_{0}^{\infty} w_{2}(y) y^{j-i} e^{-\kappa_{k} y} d y\right) x^{i-1} e^{-\kappa_{k} x} \\
= & \sum_{k=1}^{r} \sum_{i=1}^{n}\left(\sum_{j=i}^{n} \frac{B_{k j}}{(j-i) !} \int_{0}^{\infty} w_{2}(y) y^{j-i} e^{-\kappa_{k} y} d y\right) \frac{x^{i-1} e^{-\kappa_{k} x}}{(i-1) !} .
\end{aligned}
$$

Substituting all these terms in (4.5) and equating coefficients of $e^{\alpha_{i} x}$, one observes that $\alpha_{i}$ is the root of the equation (in $\xi$ )

$$
\sum_{j=1}^{n} \frac{B_{j}^{*}}{\left(\rho_{\gamma}-\xi\right)^{j}}+\sum_{i=1}^{r} \sum_{j=1}^{n} \frac{B_{i j}}{\left(\kappa_{i}+\xi\right)^{j}}=1
$$

Due to (4.1), this is equivalent to the Lundberg equation (3.25). From Appendix A, it is known that (3.25) has exactly $n$ roots with non-negative real parts. These $n$ roots are denoted by $\left\{\alpha_{i}\right\}_{i=1}^{n}$ in Section 3 (which is in accordance with the present notation). Therefore, we have that $C_{i}=0$ for $i=1,2, \ldots, n$. The remaining $n r$ roots $\left\{\alpha_{i}\right\}_{i=n+1}^{n(r+1)}$ of (3.25) have negative real parts.

Similarly, we now equate the coefficients of $x^{i-1} e^{-\kappa_{k} x}$ to arrive at

$$
\sum_{z=n+1}^{n(r+1)} C_{z} \sum_{j=i}^{n} \frac{B_{k j}}{\left(\kappa_{k}+\alpha_{z}\right)^{j-i+1}}=\sum_{j=i}^{n} \frac{B_{k j}}{(j-i) !} \int_{0}^{\infty} w_{2}(y) y^{j-i} e^{-\kappa_{k} y} d y, \quad k=1,2, \ldots, r ; i=1,2, \ldots, n
$$

Hence, (4.7) forms a system of $n r$ linear equations for $\left\{C_{i}\right\}_{i=n+1}^{n(r+1)}$, and a full characterization of $m_{\delta}(x)$ is then given by (4.6). Note that the size of the system increases with both $n$ and $r$. 
Example 4.2 When the claim size $Y$ and the observation time $T$ are both exponential (as in Example $4.1)$, with the above analysis $m_{\delta}(x)$ is reduced to

$$
m_{\delta}(x)=\left(R_{\gamma}-R_{0}\right)\left(\int_{0}^{\infty} w_{2}(y) e^{-R_{\gamma} y} d y\right) e^{-R_{0} x}, \quad x \geq 0
$$

since $\kappa_{1}=R_{\gamma}$ and $\alpha_{2}=-R_{0}$. This is consistent with the discounted density given in (2.17).

\section{$5 \quad$ Numerical illustrations}

\subsection{Comparison with the classical model}

Let us first compare the effect of random observation times (and the value of $\gamma$ ) on ruin-related quantities, in particular in comparison with the classical case of continuous observation (i.e. $\gamma \rightarrow \infty$ ). We consider the situation in Section 2.2, where the inter-observation time $T$ and the claim size $Y$ are exponentially distributed with mean $1 / \gamma$ and $1 / \nu$, respectively. Here we always assume $\nu=1, \lambda=1$ and $c=1.5$, so that the positive loading condition holds.

First, we are interested in the ruin probability $\psi(x)=\mathbb{P}(\tau<\infty \mid U(0)=x)$, which can be obtained from (2.18) by letting $\delta=0$. Figure 2 (left) shows $\psi(x)$ for various values of $\gamma$ as well as the classical case of continuous observation. The upper solid line with a discontinuity at $x=0$ corresponds to the classical ruin probability. The lower full line refers to $\gamma=1$, whereas the dotted and dashed lines are for $\gamma=5$ and $\gamma=10$, respectively. One observes that the discontinuity of the classical case at zero is smoothed out for finite values of $\gamma$, and for increasing $\gamma$ the ruin probability gets closer to the classical case as expected. Moreover, the ruin probability appears to increase with $\gamma$. This makes sense intuitively, as a larger value of $\gamma$ implies more frequent observation of the surplus process, leading to a higher chance of observing ruin.
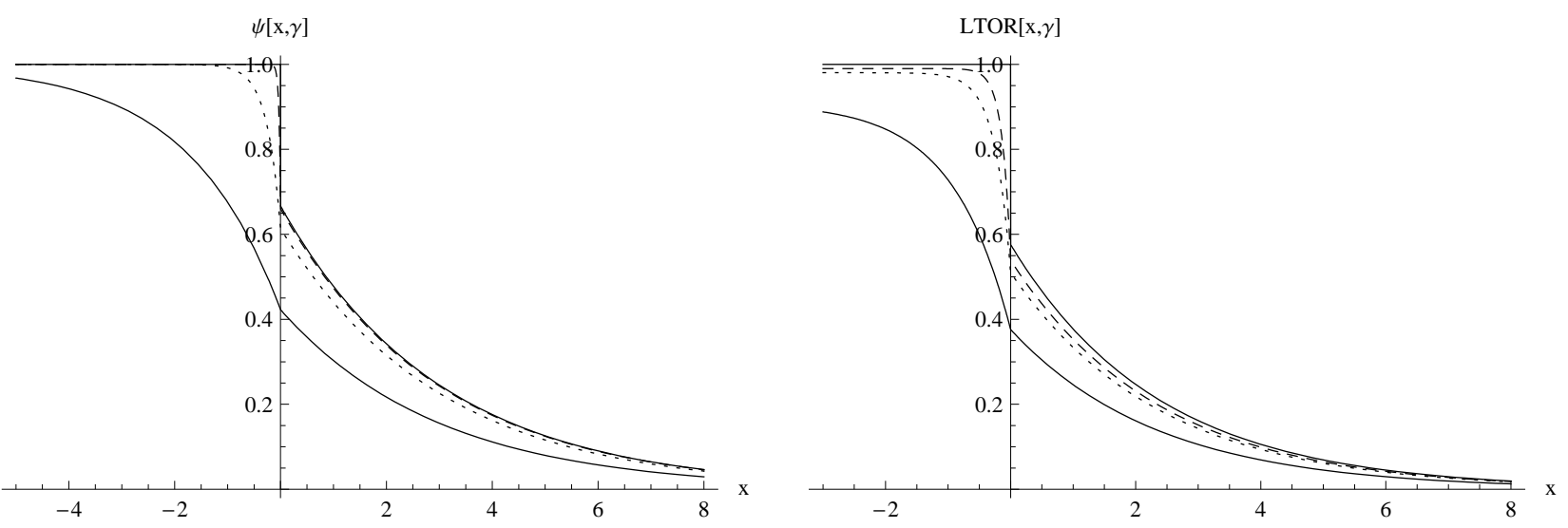

Figure 2: Ruin probability and Laplace transform of time of ruin

The Laplace transform of the time to ruin $\mathbb{E}\left[e^{-\delta \tau} I_{\{\tau<\infty\}} \mid U(0)=x\right]$ can be directly computed via (2.18). The right side of Figure 2 depicts a plot against $x$ for $\delta=0.1$. One can also see that the effect 
of a finite (but not too small) $\gamma$ in comparison to $\gamma=\infty$ is more pronounced for $\delta>0$ (right) than for $\delta=0$ (left).

The expected time of ruin $\mathbb{E}\left[\tau I_{\{\tau<\infty\}} \mid U(0)=x\right]$ can be obtained as the (negative) derivative of the Laplace transform of the time to ruin with respect to $\delta$, evaluated at $\delta=0$. The respective values as a function of $x$ are depicted in Figure 3 (with the line style as in Figure 2). Again, the discontinuity at zero is smoothed out in the random observations model.

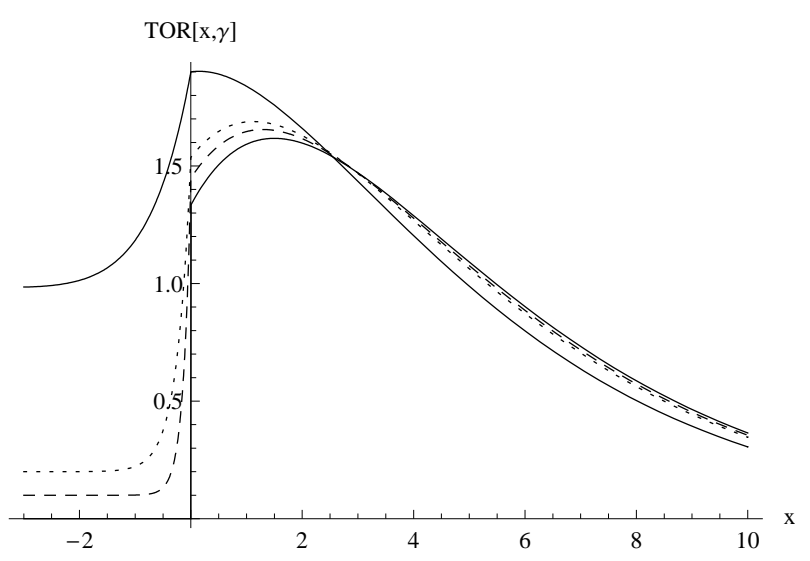

Figure 3: Expected time of ruin

The expected discounted deficit at ruin $\mathbb{E}\left[e^{-\delta \tau}\left|U\left(k^{*}\right)\right| I_{\{\tau<\infty\}} \mid U(0)=x\right]$ can be evaluated using (2.19) in Example 2.2. Figure 4 (left) plots the expected discounted deficit for $\delta=0.05$.

The joint Laplace transform of the time to ruin and the deficit at ruin $\mathbb{E}\left[e^{-\delta \tau-r_{2}\left|U\left(k^{*}\right)\right|} I_{\{\tau<\infty\}} \mid U(0)=x\right]$ is given by (2.13) and (2.14). From the densities (2.16) and (2.17) it is clear that it exists even for negative values of $r_{2}$ as long as $r_{2}>-R_{\gamma}$. For $\delta=0.05$, Figure 4 (right) and Figure 5 give the plots of the joint transform for $r_{2}=-0.5,0.5,2$. Again, the approximation of the classical solution with increasing $\gamma$ works well.
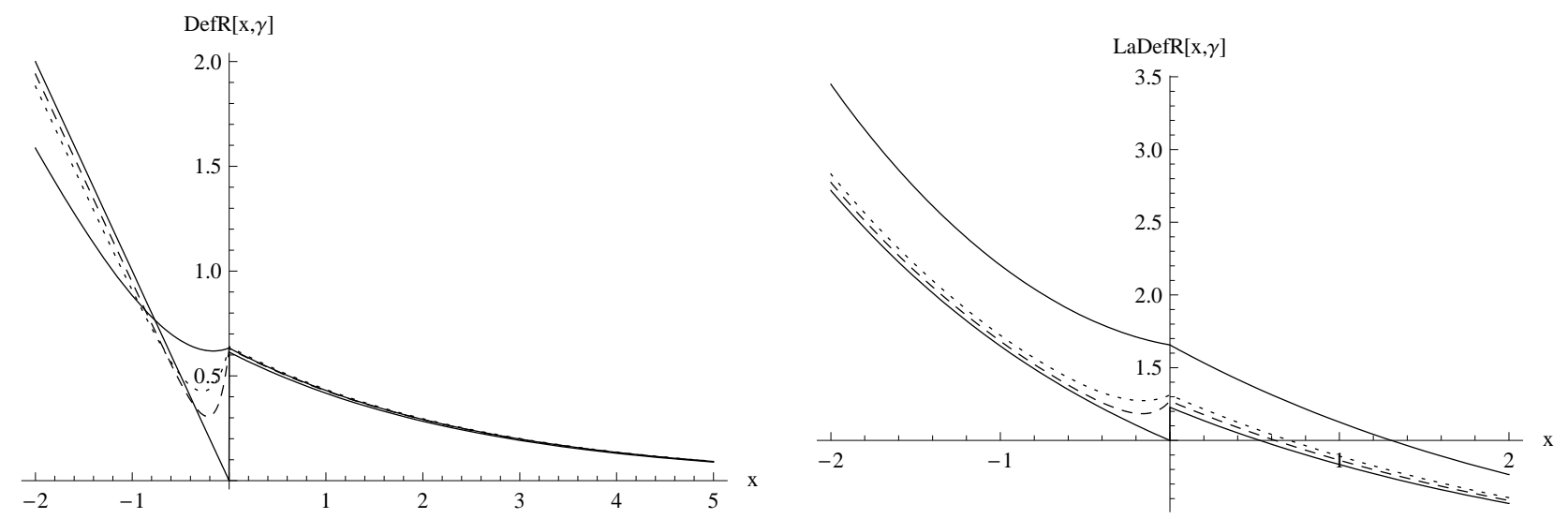

Figure 4: Expected discounted deficit at ruin and joint Laplace transform of $\left(\tau,\left|U\left(k^{*}\right)\right|\right)$ at $r_{2}=-0.5$ 

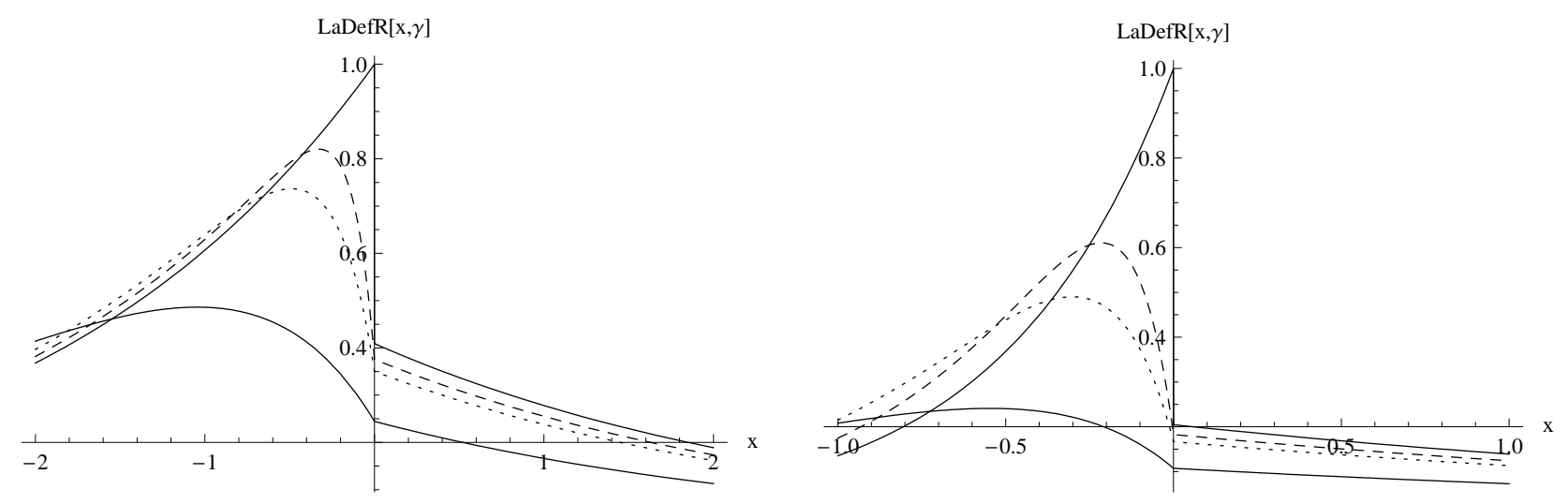

Figure 5: Joint Laplace transform of $\left(\tau,\left|U\left(k^{*}\right)\right|\right)$ at $r_{2}=0.5$ and $r_{2}=2$

\subsection{Approximating the discrete-time risk model by Erlangization}

Next, we shall compare the ruin probabilities $\psi(x)=\mathbb{P}(\tau<\infty \mid U(0)=x)$ under models with Erlang $(n)$ observation intervals for different values of $n$. The goal here is to investigate how much the values of $\psi(x)$ are affected by the 'randomness' of the observation times. This is achieved by fixing the expected time between observations while increasing the value of $n$. Note that for large values of $n$ the density of the $\operatorname{Erlang}(n)$ distribution gets more and more peaked around its mean, so that one approaches the case of deterministic periodic observation decision intervals, which is the discrete-time risk model with an underlying compound Poisson claim process. Let us fix $\lambda=1$ and $c=1.5$, and the mean of the observation intervals $\mathbb{E}[T]=2.5$, so that on average there are 2.5 claims between successive observations. Three different claim size distributions shall be considered, each of which has an expected value of 1. More specifically, we consider a sum of two exponentials with mean $1 / 3$ and $2 / 3$ (Table 1 ), an exponential claim size distribution with mean 1 (Table 2) and a mixture of two exponentials with mean 2 and $1 / 2$ (Table 3 ). The parameters are specified such that the three distributions have the same mean, but a different amount of variability (variance 0.56, 1 and 2, respectively). Since all three claim size distributions have rational Laplace transforms, the computational procedure in Section 4.2 can be used. Tables 1-3 show the respective ruin probabilities for various values of $n$ together with the ruin probability under the classical case of continuous observation. The last column gives a simulation estimate of the corresponding discrete-time risk model (using 20,000 simulation paths for each estimate) together with a $95 \%$ asymptotic confidence interval. In fact, for the given accuracy each of those simulation estimates takes several hours on a usual PC, which illustrates the advantage of the approximations obtained by the Erlangization techniques. The present approach also serves as a competitive alternative to the calculation through the usual discrete-time algorithms (see e.g. Landriault (2008)) and discretization of the claim size distribution (see De Vylder \& Goovaerts (1988) and Dickson \& Waters (1991)). One also sees that already small values of $n$ give an excellent approximation. 


\begin{tabular}{|c|c|c|c|c|c|c|c|c|c|c|}
\hline Comb Exp & Classical & $n=1$ & $n=2$ & $n=3$ & $n=4$ & $n=5$ & $n=6$ & $n=7$ & $n=15$ & Discrete (MC) \\
\hline$\psi(0)$ & 0.6667 & 0.3660 & 0.3691 & 0.3686 & 0.3679 & 0.3673 & 0.3668 & 0.3664 & 0.3651 & $\begin{array}{c}0.3689 \\
( \pm 0.0067)\end{array}$ \\
\hline$\psi(5)$ & 0.0757 & 0.0404 & 0.0429 & 0.0439 & 0.0444 & 0.0447 & 0.0449 & 0.0450 & 0.0455 & $\begin{array}{c}0.0471 \\
( \pm 0.0029)\end{array}$ \\
\hline$\psi(10)$ & 0.0083 & 0.0044 & 0.0047 & 0.0048 & 0.0049 & 0.0049 & 0.0049 & 0.0050 & 0.0050 & $\begin{array}{c}0.0042 \\
( \pm 0.0009)\end{array}$ \\
\hline$\psi(15)$ & 0.0009 & 0.0005 & 0.0005 & 0.0005 & 0.0005 & 0.0005 & 0.0005 & 0.0005 & 0.0005 & $\begin{array}{c}0.0005 \\
( \pm 0.0003)\end{array}$ \\
\hline
\end{tabular}

Table 1: $\lambda=1, c=1.5, f(x)=3 e^{-1.5 x}-3 e^{-3 x}, T \sim \operatorname{Erlang}(n)$ with $\mathbb{E}(T)=2.5$

\begin{tabular}{|c|c|c|c|c|c|c|c|c|c|c|}
\hline Exp & Classical & $n=1$ & $n=2$ & $n=3$ & $n=4$ & $n=5$ & $n=6$ & $n=7$ & $n=19$ & Discrete (MC) \\
\hline$\psi(0)$ & 0.6667 & 0.3948 & 0.4011 & 0.4022 & 0.4025 & 0.4025 & 0.4024 & 0.4023 & 0.4019 & $\begin{array}{c}0.4000 \\
( \pm 0.0068)\end{array}$ \\
\hline$\psi(5)$ & 0.1259 & 0.0746 & 0.0786 & 0.0801 & 0.0809 & 0.0814 & 0.0817 & 0.0820 & 0.0829 & $\begin{array}{c}0.0852 \\
+0.0037)\end{array}$ \\
\hline$\psi(10)$ & 0.0238 & 0.0141 & 0.0149 & 0.0152 & 0.0154 & 0.0155 & 0.0156 & 0.0156 & 0.0158 & $\begin{array}{c}0.0158 \\
(+00017)\end{array}$ \\
\hline$\psi(15)$ & 0.0045 & 0.0027 & 0.0028 & 0.0029 & 0.0029 & 0.0029 & 0.0029 & 0.0029 & 0.0030 & $\begin{array}{c}0.0029 \\
( \pm 0.0007)\end{array}$ \\
\hline
\end{tabular}

Table 2: $\lambda=1, c=1.5, f(x)=e^{-x}, T \sim \operatorname{Erlang}(n)$ with $\mathbb{E}(T)=2.5$

\begin{tabular}{|c|c|c|c|c|c|c|c|c|c|c|}
\hline Mixed Exp & Classical & $n=1$ & $n=2$ & $n=3$ & $n=4$ & $n=5$ & $n=6$ & $n=7$ & $n=14$ & Discrete (MC) \\
\hline$\psi(0)$ & 0.6667 & 0.4314 & 0.4397 & 0.4420 & 0.4431 & 0.4437 & 0.4440 & 0.4443 & 0.4450 & $\begin{array}{c}0.4536 \\
( \pm 0.0069)\end{array}$ \\
\hline$\psi(5)$ & 0.2180 & 0.1503 & 0.1569 & 0.1594 & 0.1607 & 0.1615 & 0.1620 & 0.1624 & 0.1636 & $\begin{array}{c}0.1652 \\
( \pm 0.0051)\end{array}$ \\
\hline$\psi(10)$ & 0.0783 & 0.0540 & 0.0566 & 0.0576 & 0.0581 & 0.0584 & 0.0586 & 0.0588 & 0.0593 & $\begin{array}{c}0.0609 \\
( \pm 0.0033)\end{array}$ \\
\hline$\psi(15)$ & 0.0282 & 0.0194 & 0.0204 & 0.0207 & 0.0209 & 0.0210 & 0.0211 & 0.0212 & 0.0213 & $\begin{array}{c}0.0236 \\
( \pm 0.0021)\end{array}$ \\
\hline
\end{tabular}

Table 3: $\lambda=1, c=1.5, f(x)=\frac{1}{6} e^{-0.5 x}+\frac{4}{3} e^{-2 x}, T \sim \operatorname{Erlang}(n)$ with $\mathbb{E}(T)=2.5$

In terms of the effect of randomness of observations, one observes from Tables 1-3 that, as expected, the present model under random $\operatorname{Erlang}(n)$ inter-observation times always produces lower ruin probabilities than the classical model. Moreover, as $n$ increases, the value of $\psi(x)$ increases as long as the value of the initial surplus $x$ is not too small and converges to the value of the discrete-time risk model.

Comparing the same cells across Tables 1-3, one finds that $\psi(x)$ increases with the variance of the claim size distribution, which is again in agreement with intuition.

Finally, Tables 4-6 give the expected discounted deficit at ruin for the same set of parameters. One can see that, except for small values of initial capital $x$, this quantity is not very sensitive to the value of $n$ for the used claim size distributions. 


\begin{tabular}{|c|c|c|c|c|c|c|c|c|c|c|}
\hline Comb Exp & Classical & $n=1$ & $n=2$ & $n=3$ & $n=4$ & $n=5$ & $n=6$ & $n=7$ & $n=15$ & Discrete (MC) \\
\hline$x=0$ & 0.5149 & 0.5158 & 0.5329 & 0.5399 & 0.5436 & 0.5460 & 0.5476 & 0.5488 & 0.5526 & $\begin{array}{c}0.5540 \\
( \pm 0.0157)\end{array}$ \\
\hline$x=5$ & 0.0513 & 0.0544 & 0.0569 & 0.0578 & 0.0583 & 0.0586 & 0.0588 & 0.0589 & 0.0594 & $\begin{array}{c}0.0644 \\
( \pm 0.0055)\end{array}$ \\
\hline$x=10$ & 0.0054 & 0.0058 & 0.0060 & 0.0061 & 0.0062 & 0.0062 & 0.0062 & 0.0062 & 0.0063 & $\begin{array}{c}0.0053 \\
( \pm 0.0017)\end{array}$ \\
\hline$x=15$ & 0.0006 & 0.0006 & 0.0006 & 0.0006 & 0.0007 & 0.0007 & 0.0007 & 0.0007 & 0.0007 & $\begin{array}{c}0.0006 \\
( \pm 0.0005)\end{array}$ \\
\hline
\end{tabular}

Table 4: $\mathbb{E}\left[e^{-0.005 \tau}\left|U\left(k^{*}\right)\right| I_{\{\tau<\infty\}} \mid U(0)=x\right]: \lambda=1, c=1.5, f(x)=3 e^{-1.5 x}-3 e^{-3 x}, T \sim \operatorname{Erlang}(n)$

\begin{tabular}{|c|c|c|c|c|c|c|c|c|c|c|}
\hline Exp & Classical & $n=1$ & $n=2$ & $n=3$ & $n=4$ & $n=5$ & $n=6$ & $n=7$ & $n=15$ & Discrete (MC) \\
\hline$x=0$ & 0.6602 & 0.6966 & 0.7178 & 0.7264 & 0.7310 & 0.7339 & 0.7359 & 0.7374 & 0.7431 & $\begin{array}{c}0.7449 \\
( \pm 0.0200) \\
0.1396 \\
( \pm 0.0090) \\
0.0254 \\
( \pm 0.0038) \\
x=10\end{array}$ \\
0.1207 & 0.1274 & 0.1318 & 0.1334 & 0.1343 & 0.1348 & 0.1352 & 0.1355 & 0.1365 & 0.0037 \\
$x=15$ & 0.0040 & 0.0043 & 0.0044 & 0.0045 & 0.0045 & 0.0045 & 0.0045 & 0.0045 & 0.0046 & $\begin{array}{c}0.0013) \\
( \pm 0.0241\end{array}$ \\
\hline
\end{tabular}

Table 5: $\mathbb{E}\left[e^{-0.005 \tau}\left|U\left(k^{*}\right)\right| I_{\{\tau<\infty\}} \mid U(0)=x\right]: \lambda=1, c=1.5, f(x)=e^{-x}, T \sim \operatorname{Erlang}(n)$

\begin{tabular}{|c|c|c|c|c|c|c|c|c|c|c|}
\hline Mixed Exp & Classical & $n=1$ & $n=2$ & $n=3$ & $n=4$ & $n=5$ & $n=6$ & $n=7$ & $n=15$ & Discrete (MC) \\
\hline$x=0$ & 0.9825 & 1.1222 & 1.1521 & 1.1642 & 1.1707 & 1.1747 & 1.1775 & 1.1795 & 1.1855 & $\begin{array}{c}1.1737 \\
( \pm 0.0297) \\
0.4122 \\
\pm 0.019) \\
x=5\end{array}$ \\
$x=10$ & 0.3918 & 0.3980 & 0.4071 & 0.4103 & 0.4119 & 0.4129 & 0.4136 & 0.4141 & 0.4156 & $\begin{array}{c}0.1453 \\
( \pm 0.0115) \\
0.05652 \\
( \pm 0.0074)\end{array}$ \\
\hline
\end{tabular}

Table 6: $\mathbb{E}\left[e^{-0.005 \tau}\left|U\left(k^{*}\right)\right| I_{\{\tau<\infty\}} \mid U(0)=x\right]: \lambda=1, c=1.5, f(x)=\frac{1}{6} e^{-0.5 x}+\frac{4}{3} e^{-2 x}, T \sim \operatorname{Erlang}(n)$

\subsection{The last observed reserve before ruin}

Finally, let us consider the surplus prior to ruin $U\left(k^{*}-1\right)$ or, equivalently, the last observed reserve before ruin. We assume exponentially distributed inter-observation time $T$ and claim size $Y$, with parameters $\nu=1, \lambda=1$ and $c=1.5$. The Laplace transform $\mathbb{E}\left[e^{-r_{1} U\left(k^{*}-1\right)} I_{\{\tau<\infty\}} \mid U(0)=x\right]$ of $U\left(k^{*}-1\right)$ can be retrieved from (4.4) by letting $\delta=0$ and $w_{2}(.) \equiv 1$. Figure 6 (left) shows its plot against $x$ for $r_{1}=0.5$. The upper solid line corresponds to the classical model, whereas the lower solid line corresponds to $\gamma=1$. The dotted and dashed lines represent again $\gamma=5$ and $\gamma=10$, respectively. The corresponding expected value $\mathbb{E}\left[U\left(k^{*}-1\right) I_{\{\tau<\infty\}} \mid U(0)=x\right]$ of $U\left(k^{*}-1\right)$ is given on the right-hand side of Figure 6 . One can again observe the rate at which the values approach the one of the classical continuous-time model as $\gamma$ gets larger. 

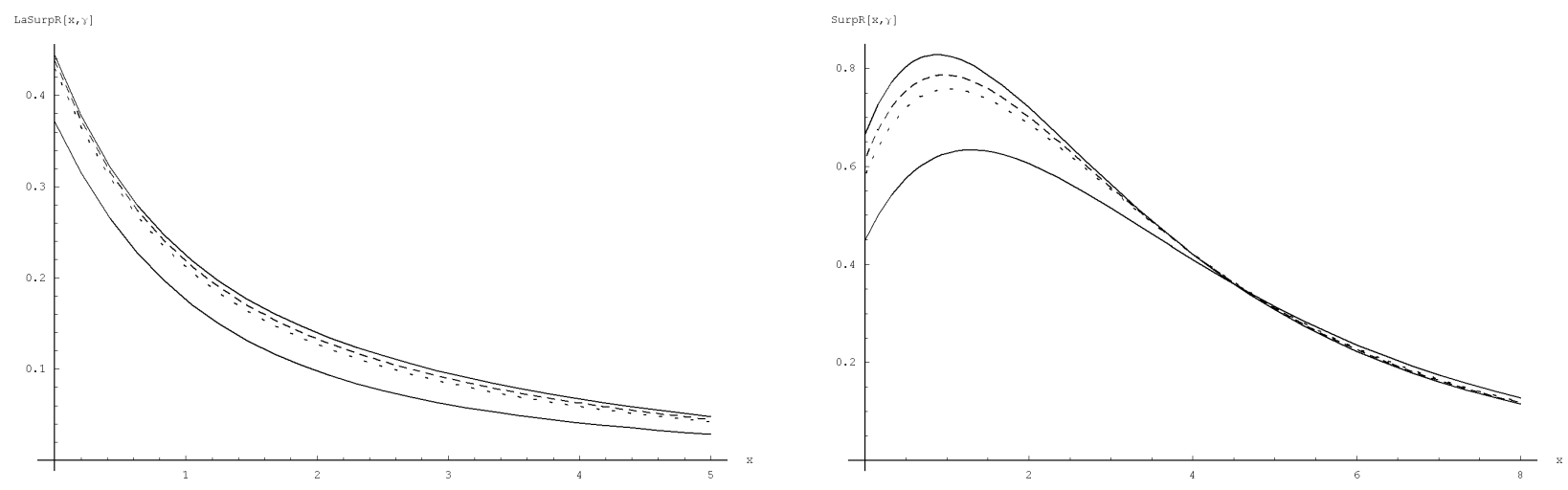

Figure 6: Laplace transform of $U\left(k^{*}-1\right)$ at $r_{1}=0.5$ and expected value of $U\left(k^{*}-1\right)$

Acknowledgments: Hansjörg Albrecher and Stefan Thonhauser gratefully acknowledge support from the Swiss National Science Foundation Project 200021-124635/1. Eric C.K. Cheung acknowledges a start-up fund provided by the Faculty of Science and the Department of Statistics and Actuarial Science as well as the Seed Funding for Basic Research (Project number: 201103159001) provided by the University Research Committee at the University of Hong Kong.

\section{References}

[1] H. Albrecher, E.C.K. Cheung and S. Thonhauser. 2010. Randomized observation periods for the compound Poisson risk model: Dividends. ASTIN Bulletin, in press.

[2] S. Asmussen and H. Albrecher. 2010. Ruin Probabilities. Second Edition, World Scientific, New Jersey.

[3] S. Asmussen, F. Avram and M. Usabel. 2002. Erlangian approximations for finite-horizon ruin probabilities. ASTIN Bulletin 32(2): 267-281.

[4] A.L. Badescu, E.C.K. Cheung and D. Landriault. 2009. Dependent risk models with bivariate phase-type distributions. Journal of Applied Probability 46(1): 113-131.

[5] E.C.K. Cheung, D. Landriault, G.E. Willmot and J.-K. Woo. 2010a. Gerber-Shiu analysis with a generalized penalty function. Scandinavian Actuarial Journal 2010(3): 185-199.

[6] E.C.K. Cheung, D. Landriault, G.E. Willmot and J.-K. Woo. 2010b. Structural properties of Gerber-Shiu functions in dependent Sparre Andersen models. Insurance: Mathematics and Economics 46(1): 117-126.

[7] F. De Vylder and M.J. Goovaerts. 1988. Recursive calculation of finite-time ruin probabilities. Insurance: Mathematics and Economics 7(1): 1-7.

[8] D.C.M. Dickson And C. Hipp. 2001. On the time to ruin for Erlang(2) risk processes. Insurance: Mathematics and Economics 29(3): 333-344. 
[9] D.C.M. Dickson And H.R. Waters. 1991. Recursive calculation of survival probabilities. ASTIN Bulletin 21(2): 199-221.

[10] H.U. Gerber And E.S.W. Shiu. 1998. On the time value of ruin. North American Actuarial Journal 2(1): 48-72.

[11] D. LAndRIAUlt. 2008. On a generalization of the expected discounted penalty function in a discretetime insurance risk model. Applied Stochastic Models in Business and Industry 24(6): 525-539.

[12] S. Li And J. GarRIDo. 2004. On ruin for the $\operatorname{Erlang}(n)$ risk process. Insurance: Mathematics and Economics 34(3): 391-408.

[13] S. Li and J. Garrido. 2005. On a general class of risk processes: Analysis of the Gerber-Shiu function. Advances in Applied Probability 37(3): 836-856.

[14] V. Ramaswami, D.G. Woolford and D.A. Stanford. 2008. The Erlangization method for Markovian fluid flows. Annals of Operations Research 160(1): 215-225.

[15] D.A. Stanford, F. Avram, A.L. Badescu, L. Breuer, A. Da Silva Soares and G. LaTOUCHE. 2005. Phase-type approximations to finite-time ruin probabilities in the Sparre-Anderson and stationary renewal risk models. ASTIN Bulletin 35(1): 131-144.

[16] D.A. Stanford, K. Yu and J. Ren. 2011. Erlangian approximation to finite time ruin probabilities in perturbed risk models. Scandinavian Actuarial Journal, No.1, 38-58.

[17] G.E. Willmot. 2007. On the discounted penalty function in the renewal risk model with general interclaim times. Insurance: Mathematics and Economics 41(1): 17-31.

[18] G.E. Willmot and X.S. Lin. 2001. Lundberg Approximations for Compound Distributions with Applications. New York: Springer-Verlag.

\section{A Application of Rouché's Theorem}

The purpose of this section is to show that the Lundberg equation (3.25) has exactly $n$ roots with non-negative real part. Using (3.2), one can rewrite (3.25) as

$$
\left(\frac{\gamma}{\gamma+\delta-c \xi+\lambda\left[1-\widetilde{f}_{Y}(\xi)\right]}\right)^{n}=1,
$$

By De Moivre's formula, the roots of the above equation are equivalent to those of the set of $n$ equations

$$
\gamma+\delta-c \xi+\lambda\left[1-\widetilde{f}_{Y}(\xi)\right]=\gamma\left(\cos \frac{2 k \pi}{n}+i \sin \frac{2 k \pi}{n}\right), \quad k=0,1, \ldots, n-1 .
$$

First, note that for $k=0$ the above equation reduces to (3.8) with $\gamma=0$, which is well-known to have a unique non-negative root under $\delta>0$ or $c>\lambda \mathbb{E}[Y]$. Thus, we can restrict the focus to the cases $k=1,2, \ldots, n-1$ in (A.1). 
We aim to show that for each fixed $k=1,2, \ldots, n-1$, the equation (A.1) has a unique root with positive real part. It will be helpful to write (A.1) in the form of

$$
\varsigma_{1, k}(\xi)=\varsigma_{2}(\xi),
$$

where

$$
\varsigma_{1, k}(\xi)=\frac{\lambda+\delta+\gamma\left(1-\cos \frac{2 k \pi}{n}\right)}{c}-i\left(\frac{\gamma}{c} \sin \frac{2 k \pi}{n}\right)-\xi \quad \text { and } \quad \varsigma_{2}(\xi)=\frac{\lambda}{c} \widetilde{f}_{Y}(\xi) .
$$

To apply Rouché's Theorem, for $\epsilon>0$ we consider the contour $C_{\epsilon}$ on the complex plane consisting of (i) the semi-circle of radius $\epsilon$ running clockwise from $i \epsilon$ to $-i \epsilon$; and (ii) the part of the imaginary axis running from $-i \epsilon$ to $i \epsilon$. For $\xi$ on the semi-circle, one has $|\xi|=\epsilon$ and $\left|\widetilde{f}_{Y}(\xi)\right| \leq \widetilde{f}_{Y}(|\xi|)=\widetilde{f}_{Y}(\epsilon) \leq 1$, and hence

$$
\left|\varsigma_{1, k}(\xi)\right|>\left|\varsigma_{2}(\xi)\right|
$$

for $\epsilon$ sufficiently large. Next, for $\xi$ on the part of imaginary axis of interest, one has the parametric expression $\xi=i a \epsilon$ for $-1 \leq a \leq 1$. Then

$$
\begin{aligned}
\left|\varsigma_{1, k}(i a \epsilon)\right| & =\left|\frac{\lambda+\delta+\gamma\left(1-\cos \frac{2 k \pi}{n}\right)}{c}-i\left(\frac{\gamma}{c} \sin \frac{2 k \pi}{n}-a \epsilon\right)\right| \\
& \geq \frac{\lambda+\delta+\gamma\left(1-\cos \frac{2 k \pi}{n}\right)}{c} \\
& >\frac{\lambda}{c} \\
& \geq\left|\varsigma_{2}(i a \epsilon)\right|,
\end{aligned}
$$

where the second last line of strict inequality follows from the fact that $1-\cos (2 k \pi / n)>0$ for $k=$ $1,2, \ldots, n-1$. Therefore we have established $\left|\varsigma_{1, k}(\xi)\right|>\left|\varsigma_{2}(\xi)\right|$ on the entire contour $C_{\epsilon}$ for $\epsilon$ sufficiently large. Now for $\epsilon \rightarrow \infty$, the assertion follows from Rouché 's Theorem.

\section{B Convergence to the classical risk model for $\gamma \rightarrow \infty$}

Let $n=1$. First we will show (3.28). Due to (3.19), it is sufficient to show that

$$
\lim _{\gamma \rightarrow \infty} g_{\delta,+}(y)=0 \quad \text { for almost all } y>0 .
$$

Using expression (3.18), we consider the Laplace transform

$$
\begin{aligned}
\widetilde{g}_{\delta,+}(s) & =\frac{\gamma}{c} \sum_{k=1}^{\infty} \phi^{k} \mathcal{T}_{s} \mathcal{T}_{\rho_{\gamma}} f_{L}^{* k}(0)=\frac{\gamma}{c} \sum_{k=1}^{\infty} \phi^{k}\left(\frac{\left[\widetilde{f}_{L}(s)\right]^{k}-\left[\widetilde{f}_{L}\left(\rho_{\gamma}\right)\right]^{k}}{\rho_{\gamma}-s}\right) \\
& =\frac{\gamma}{c\left(\rho_{\gamma}-s\right)}\left(\frac{\phi \widetilde{f}_{L}(s)}{1-\phi \widetilde{f}_{L}(s)}-\frac{\phi \widetilde{f}_{L}\left(\rho_{\gamma}\right)}{1-\phi \widetilde{f}_{L}\left(\rho_{\gamma}\right)}\right) .
\end{aligned}
$$

Since $\rho_{\gamma}>0$ is the unique positive root of (3.8), one observes that $\lim _{\gamma \rightarrow \infty} \rho_{\gamma}=\infty$ and $\lim _{\gamma \rightarrow \infty} \gamma /\left(c \rho_{\gamma}\right)=$ 1 , and hence from (3.10) that $\lim _{\gamma \rightarrow \infty} \phi=0$. Thus $\lim _{\gamma \rightarrow \infty} \widetilde{g}_{\delta,+}(s)=0$ and consequently (B.1) holds, verifying (3.28). 
Next, we shall study the limit of the discounted density $h_{2, \delta}^{*}(z, y \mid 0)$. From $(3.27)$, one has that

$$
h_{2, \delta}^{*}(z, y \mid 0)=e^{-\rho_{0} z}\left[\rho_{\gamma} g_{\delta,+}(z+y)-\rho_{0} g_{\delta,+}(z+y)\right], \quad z, y>0,
$$

since $\alpha_{1}=\rho_{0}$ when $n=1$. As $\rho_{0}$ does not depend on $\gamma$, the second term in the above equation converges to 0 for almost all $z, y>0$ as $\gamma \rightarrow \infty$. For the first term we have from (B.2)

$$
\rho_{\gamma} \widetilde{g}_{\delta,+}(s)=\frac{\gamma}{c\left(\rho_{\gamma}-s\right)}\left(\frac{\rho_{\gamma} \phi \widetilde{f}_{L}(s)}{1-\phi \widetilde{f}_{L}(s)}-\frac{\rho_{\gamma} \phi \widetilde{f}_{L}\left(\rho_{\gamma}\right)}{1-\phi \widetilde{f}_{L}\left(\rho_{\gamma}\right)}\right) .
$$

The factor in front of the bracket tends to 1 , and in view of (3.11) the first term inside behaves like

$$
\frac{\rho_{\gamma} \phi \widetilde{f}_{L}(s)}{1-\phi \widetilde{f}_{L}(s)}=\frac{\rho_{\gamma} \frac{\lambda}{c} \frac{\tilde{f}_{Y}(s)-\tilde{f}_{Y}\left(\rho_{\gamma}\right)}{\rho_{\gamma}-s}}{1-\frac{\lambda}{c} \frac{\widetilde{f}_{Y}(s)-\widetilde{f}_{Y}\left(\rho_{\gamma}\right)}{\rho_{\gamma}-s}} \rightarrow \frac{\lambda}{c} \widetilde{f}_{Y}(s) \quad \text { as } \gamma \rightarrow \infty .
$$

For the second term we have

$$
\begin{aligned}
\frac{\rho_{\gamma} \phi \widetilde{f}_{L}\left(\rho_{\gamma}\right)}{1-\phi \widetilde{f}_{L}\left(\rho_{\gamma}\right)} & =\frac{\lambda}{c} \frac{\rho_{\gamma}}{1-\phi \widetilde{f}_{L}\left(\rho_{\gamma}\right)} \mathcal{T}_{\rho_{\gamma}}^{2} f_{Y}(0)=\frac{\lambda}{c} \frac{\rho_{\gamma}}{1-\phi \widetilde{f}_{L}\left(\rho_{\gamma}\right)} \int_{0}^{\infty} y e^{-\rho_{\gamma} y} f_{Y}(y) d y \\
& \leq \frac{\lambda}{c} \frac{\rho_{\gamma}}{1-\phi \widetilde{f}_{L}\left(\rho_{\gamma}\right)}\left(\sup _{x>0} f_{Y}(x)\right) \int_{0}^{\infty} y e^{-\rho_{\gamma} y} d y \\
& =\frac{\lambda}{c \rho_{\gamma}} \frac{1}{1-\phi \widetilde{f}_{L}\left(\rho_{\gamma}\right)}\left(\sup _{x>0} f_{Y}(x)\right) \\
& \rightarrow 0 \quad \text { as } \gamma \rightarrow \infty
\end{aligned}
$$

under the mild assumption on the claim size density

$$
\sup _{x>0} f_{Y}(x)<\infty
$$

Combining the above results gives (3.29) under the assumption (B.3). 